\title{
Heterologous prime-boost vaccination protects against EBV antigen-expressing lymphomas
}

\author{
Julia Rühl, ${ }^{1}$ Carmen Citterio, ${ }^{1}$ Christine Engelmann, ${ }^{1}$ Tracey Haigh, ${ }^{2}$ Andrzej Dzionek, ${ }^{3}$ Johannes Dreyer, ${ }^{4}$ Rajiv Khanna, ${ }^{5}$ \\ Graham S. Taylor, ${ }^{2}$ Joanna B. Wilson, ${ }^{6}$ Carol S. Leung, ${ }^{7}$ and Christian Münz ${ }^{1}$

\begin{abstract}
'Viral Immunobiology, Institute of Experimental Immunology, University of Zurich, Zurich, Switzerland. ²ancer Immunology and Immunotherapy Centre, University of Birmingham, Birmingham, United Kingdom. ${ }^{3}$ Miltenyi Biotec GmbH, Bergisch Gladbach, Germany. ${ }^{4}$ Institute for Pathology, Unfallkrankenhaus Berlin, Berlin, Cermany. ${ }^{5}$ IMIMR Berghofer Centre for Immunotherapy and Vaccine Development and Tumour Immunology Laboratory, Department of Immunology, QIMR Berghofer Medical Research Institute, Brisbane, Australia. ${ }^{6}$ College of Medical, Veterinary and Life Sciences, University of Glasgow, Glasgow, United Kingdom. University of Oxford, Nuffield Department of Medicine, Ludwig Institute for Cancer Research, Oxford, United Kingdom.
\end{abstract}

\begin{abstract}
The Epstein-Barr virus (EBV) is one of the predominant tumor viruses in humans, but so far no therapeutic or prophylactic vaccination against this transforming pathogen is available. We demonstrated that heterologous prime-boost vaccination with the nuclear antigen 1 of EBV (EBNA1), either targeted to the DEC205 receptor on DCs or expressed from a recombinant modified vaccinia virus Ankara (MVA) vector, improved priming of antigen-specific $C D 4^{+} \mathrm{T}$ cell help. This help supported the expansion and maintenance of EBNA1-specific $\mathrm{CD8}^{+} \mathrm{T}$ cells that are most efficiently primed by recombinant adenoviruses that encode EBNA1. These combined $C D 4^{+}$and $C D 8^{+} \mathrm{T}$ cell responses protected against EBNA1-expressing $\mathrm{T}$ and $\mathrm{B}$ cell lymphomas, including lymphoproliferations that emerged spontaneously after EBNA1 expression. In particular, the heterologous EBNA1-expressing adenovirus, boosted by EBNA1-encoding MVA vaccination, demonstrated protection as a prophylactic and therapeutic treatment for the respective lymphoma challenges. Our study shows that such heterologous prime-boost vaccinations against EBV-associated malignancies as well as symptomatic primary EBV infection should be further explored for clinical development.
\end{abstract}

\section{Introduction}

Epstein-Barr virus (EBV) is one of the most successful human pathogens, establishing persistent infection in more than $95 \%$ of adults (1). At the same time, this common $\gamma$-herpesvirus is the most growth-transforming pathogen in vitro and is associated with a variety of B cell lymphomas and epithelial cell carcinomas in vivo (2). These amount to approximately 200,000 new cancers every year, therefore, EBV constitutes an important target for therapeutic intervention (3). The viral tumorigenic potential is primarily due to the latent EBV infection programs, which express up to 8 proteins and more than 40 nontranslated RNAs (1). Together with these nontranslated RNAs, the 6 EBV nuclear antigens (EBNAs) and 2 latent membrane proteins (LMPs) of the latency III program transform B cells in vitro into lymphoblastoid cell lines (LCLs) and are found in non-Hodgkin's lymphomas like post-transplantation lymphoproliferative disease (PTLD), immunoblastic lymphomas, and diffuse large B cell lymphomas (DLBCLs) of immuno-

\section{Related Commentary: p. 1836}

Authorship note: CSL and CM contributed equally to this work. Conflict of interest: $A D$ is an employee of Miltenyi Biotec $\mathrm{GmbH}$. RK is a consultant and member of the scientific advisory board of Atara Biotherapeutics and has received a license fee payment and research funding from Atara Biotherapeutics. Copyright: @ 2019, The American Society for Clinical Investigation.

Submitted: October 5, 2018; Accepted: March 5, 2019; Published: April 15, 2019

Reference information: / Clin Invest. 2019;129(5):2071-2087.

https://doi.org/10.1172/JCl125364. compromised patients (2). EBNA1, LMP1, and LMP2 expression, the more restricted latency II program, is characteristic for EBVassociated Hodgkin's lymphoma, extranodal NK/T cell lymphomas, nasopharyngeal carcinoma, and gastric carcinoma. Finally, Burkitt's lymphomas often express only EBNA1 as the sole EBV protein. Interestingly, all these EBV latency programs are already present in healthy EBV carriers in distinct differentiation stages of infected B cells (4), and EBV seems to persist long term in memory B cells without any viral protein expression (5). The presence of growth-transforming latent EBV expression in healthy virus carriers and the increased incidence of B cell lymphomas of all EBV latency programs in patients with primary immunodeficiencies or immune-suppressive HIV coinfection $(6,7)$ suggest that asymptomatic, persistent EBV infection relies on comprehensive immune control of all latency patterns.

Indeed, the list of primary immunodeficiencies that predispose individuals to EBV-associated diseases identifies cytotoxic lymphocytes as the cornerstone of this immune control $(6,8)$. More specifically, mutations in $\mathrm{T}$ cell receptor signaling identify conventional $\alpha \beta$ T cells and innate NKT as well as $\gamma \delta$ T cells as components of this immune control $(9,10)$. Among these, conventional $\alpha \beta$ T cells have been used therapeutically after expansion with LCLs or defined EBV antigens primarily for the treatment of PTLDs (11). While the antigen specificities of these clinically efficacious $\mathrm{T}$ cell transfers often remain undefined, EBNA1 has at least been identified as one of the protective EBV antigens by the treatment success seen after adoptive transfer of $\mathrm{T}$ cell populations that have been selected via 
A

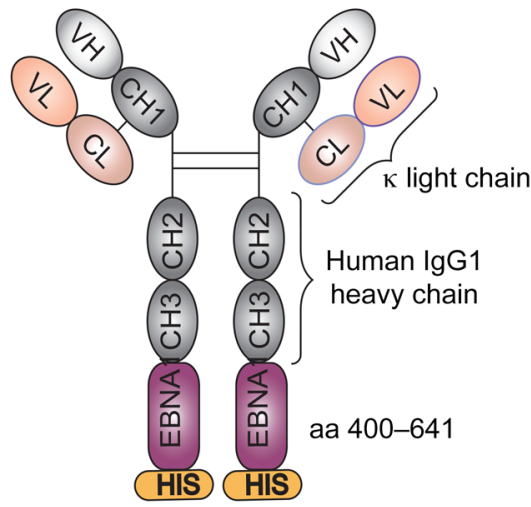

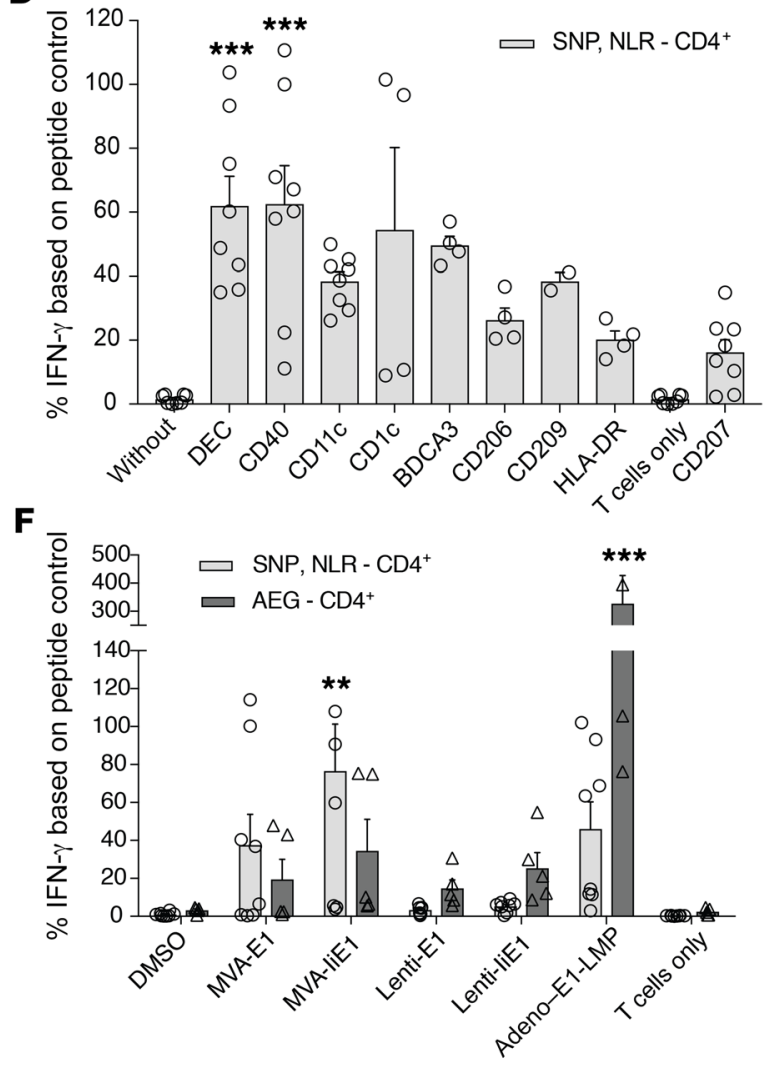

B

C

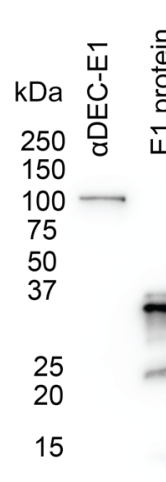

$\mathrm{kDa}$

75

50
37

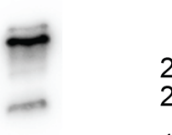

20

15

Actin

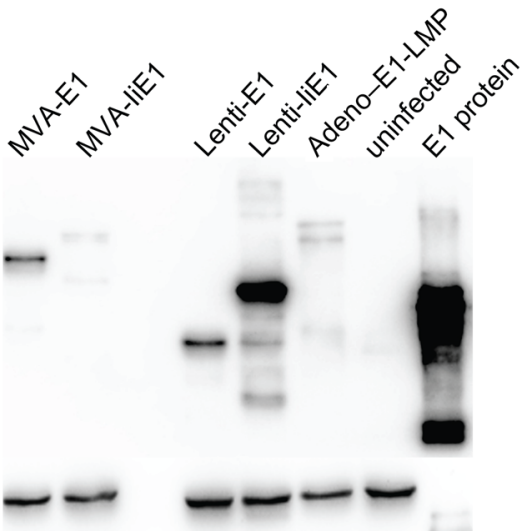

E

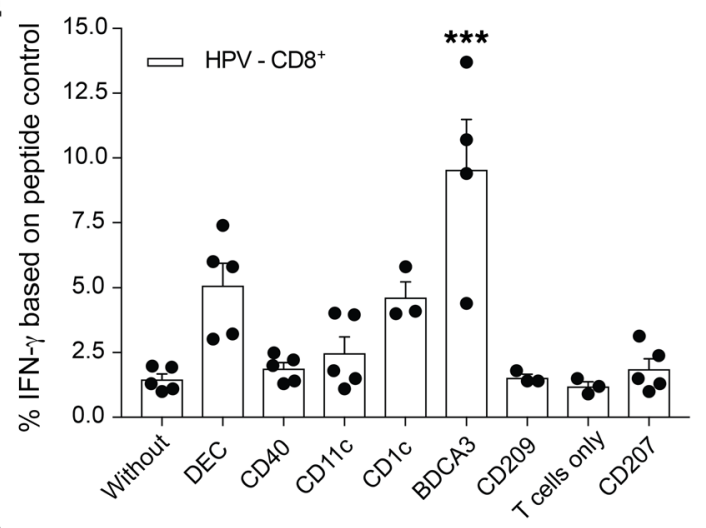

G

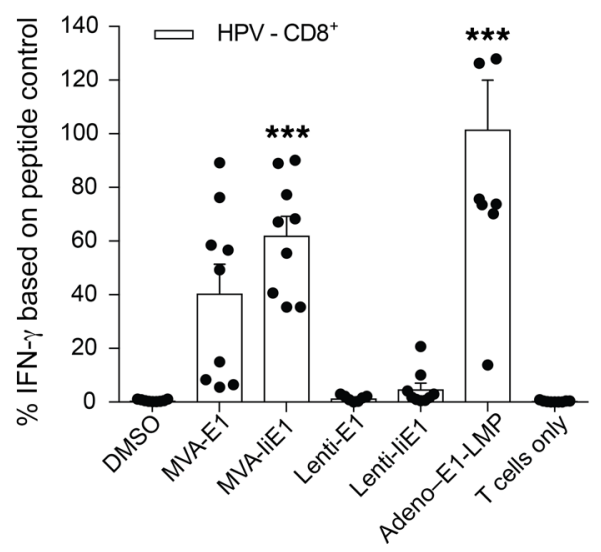

Figure 1. Human $\mathrm{CD4}^{+}$and $\mathrm{CD8}^{+} \mathrm{T}$ cell recognition of EBNA1 carrying or encoding vaccine formulations. (A) Structure of humanized EBNA1-Ab fusion proteins. (B) Western blot analysis of human $\alpha$ DEC205-EBNA1 Ab under reducing conditions using rat $\alpha E B N A 1$ Ab (clone $1 \mathrm{H} 4$ ). Lane 1 represents heavychain EBNA1 (100 kDa) and lane 2 recombinant truncated EBNA1. (C) Western blot analysis of viral vectors encoding truncated EBNA1, using rat $\alpha$ EBNA1 Ab (clone 1H4) 48 hours after infection of HEK293T cells. MVA-E1 carries EBNA1 without the Gly/Ala repeat and runs at approximately $45 \mathrm{kDa}$ (25); MVA-liE1 has the additional invariant chain domain. Lenti-E1 carries only EBNA1 from aa 400-641 with an approximate size of 30 kDa. Infection with Adeno-E1-LMP also leads to expression of the Gly/Ala repeat-deleted EBNA1 protein, however with additional LMP polyepitopes (26), and migrates at approximately 60 kDa. Lane 6 represents uninfected HEK293T cells and lane 7 recombinant truncated EBNA1. (D and E) Autologous PBMCs were incubated with medium for 4 hours with $1 \mu \mathrm{g} / \mathrm{ml}$ EBNA1 fused to an Ab against the indicated receptors, or for 1 hour with the cognate peptides for the respective T cell clones. Coculture with (D) EBNA1-specific CD4+ $\mathrm{T}$ cell clones, with cognate epitope NLR and SNP shown in the gray bars, and (E) EBNA1-specific CD8 ${ }^{+}$T cell clones, with cognate epitope HPV shown in the white bars. T cell activity was measured by IFN- $\gamma$ release into the supernatant. IFN- $\gamma$ signal is shown as a percentage of the peptide control. Data are shown as the mean \pm SD of at least 2 independent experiments. ${ }^{* * *} P<0.005$ versus unspecific CD207-targeting; 1 -way ANOVA with Bonferroni's pre-test . (F and G) Autologous PBMCs were infected with DMSO control, MVA-EBNA1, MVA-liEBNA1, or Adeno-EBNA1-LMP at a MOI of 10 for 48 hours and with Lenti-EBNA1 or Lenti-liEBNA1 for 96 hours. Coculture with (F) EBNA1-specific CD4+ T cell clones, with cognate epitope NLR and SNP shown in the light gray bars and cognate epitope AEG shown in the dark gray bars, and (G) EBNA1-specific CD8 ${ }^{+} \mathrm{T}$ cell clones, with cognate epitope HPV shown in the white bars. T cell activity was determined as in $\mathbf{D}$ and $\mathbf{E}$. Data are shown as the mean \pm SD of 2 independent experiments. ${ }^{* *} P<0.01$ and ${ }^{*}{ }^{*} P<0.005$; 1 -way ANOVA plus Bonferroni's pre-test. 
cytokine production in response to this latent EBV antigen (12). Furthermore, EBNA1 is consistently recognized at least by $\mathrm{CD} 4^{+} \mathrm{T}$ cells in nearly all healthy EBV carriers $(13,14)$, and both EBNA1-specific $\mathrm{CD}^{+}$and $\mathrm{CD}^{+} \mathrm{T}$ cells are able to target EBV-transformed $\mathrm{B}$ cells (15-17). For the direct recognition of EBV-transformed B cells by EBNA1-specific $\mathrm{CD} 4^{+} \mathrm{T}$ cells, it has been demonstrated that this antigen is intracellularly processed for MHC class II presentation via macroautophagy $(18,19)$. Finally, EBNA1 is also an attractive target, because it is the sole EBV protein that is expressed in all EBV-associated malignancies and can therefore serve as a viral tumor antigen to be targeted by passive and active vaccination against EBV-associated diseases (20). Thus, we aimed to identify a potent vaccine formulation to prime EBNA1-specific $\mathrm{CD} 4^{+}$and $\mathrm{CD}^{+} \mathrm{T}$ cell responses. Our findings suggest that heterologous prime-boost vaccinations for $\mathrm{CD} 4^{+} \mathrm{T}$ cell priming by either recombinant Abs that target EBNA1 to DCs using DEC205 ( $\alpha$ DEC-E1) or MVA-encoding EBNA1 (MVA-E1) need to be combined with CD8 ${ }^{+}$ T cell priming by EBNA1-encoding adenovirus (Adeno-E1-LMP) to establish efficient long-term immune control of EBNA1-expressing lymphomas. This immune control relied on both $\mathrm{CD}^{+}$and $\mathrm{CD} 8^{+}$ $\mathrm{T}$ cell populations, which reached the highest cytotoxic $\mathrm{CD} 8^{+} \mathrm{T}$ cell frequencies and maintained a broad repertoire of $\mathrm{CD}^{+} \mathrm{T}$ cell effector functions only in the presence of $\mathrm{CD}^{+} \mathrm{T}$ cell help. We propose that our most successful prime-boost regimens ( $\alpha$ DEC-E1 plus Adeno-E1-LMP or Adeno-E1-LMP plus MVA-IiE1) should be further developed for clinical application.

\section{Results}

Human $C D 4^{+}$and $C D 8^{+} T$ cell recognition of EBNA1 carrying or encoding vaccine formulations. It has been previously demonstrated that targeting antigens to the type I transmembrane multilectin receptor DEC2O5 that is preferentially expressed on conventional type $1 \mathrm{DCs}(\mathrm{cDC1s})$ leads to prominent $\mathrm{CD} 4^{+} \mathrm{T}$ cell responses but has only a subtle effect on $\mathrm{CD}^{+} \mathrm{T}$ cell induction in vitro and in vivo (21-24). To potentially identify a more suitable receptor for enhanced antigen delivery to both MHC class I and II pathways, we constructed fusion proteins of EBNA1 and Abs directed at 9 other receptors with different internalization kinetics and expressed by similar or different myeloid cell subsets (as indicated in parentheses): BDCA3 (cDC1s), CD206 (monocytes), CD207 (Langerhans cells and dermal cDC1s), CD209 (DCs), CD40 (all antigen-presenting cells), HLA-DR (all antigen-presenting cells), CD1c (cDC2s), and CD11c (in blood, primarily DCs).

In the first step toward the generation of EBNA1-Ab fusion proteins, the variable region sequences of the chosen Abs were selected from mouse hybridoma cell lines. The sequenced variable regions of the heavy and light chains were synthesized into HEK expression vectors, and the sequence coding for EBNA1 aa 400-641 was added to the heavy chain vector.

The EBNA1-Ab fusion proteins that were produced consisted of human constant regions including $\kappa$ light chain and IgG1 heavy chain, the EBNA1 ${ }_{400-641}$ sequence, and a His-tag for easier detection and purification (Figure 1A). The fusion Abs only differ in their variable regions. EBNA1-Ab fusion proteins were produced in stable infected human embryonic kidney 293 T cell (HEK293T cell) lines, and their purification was monitored by SDS-PAGE and EBNA1 Western blotting, with which the heavy chain can be detected at an apparent weight of approximately $100 \mathrm{kDa}$ (Figure 1B, Supplemental Figure 1B; supplemental material available online with this article; https://doi.org/10.1172/JCI125364DS1). Binding specificity after cloning was confirmed through a competitive binding assay, in which binding of the original hybridoma Abs on a target cell could be overcome by prior incubation with the engineered Ab constructs (Supplemental Figure 1A). EBNA1-Abs for all 8 receptors and for DEC205 were produced and maintained their receptor binding activity.

To assess the MHC class I and II presentation of these receptor-targeted EBNA1-Abs, we generated EBNA1-specific CD4 ${ }^{+}$and $\mathrm{CD}^{+} \mathrm{T}$ cell clones from healthy EBV carriers. We used $\mathrm{CD} 4^{+} \mathrm{T}$ cell clones recognizing different epitopes, designated SNP restricted through HLA-DR51, NLR restricted through HLA-DR1, and AEG restricted through HLA-DQ2/3. In addition, we used established EBNA1-specific CD8 ${ }^{+} \mathrm{T}$ cell clones that were specific for the HPV epitope restricted through HLA-B35, because this specificity can be readily cloned from HLA-B35-positive EBV carriers. PBMCs were incubated with $1 \mu \mathrm{M}$ EBNA1 fusion Abs for 4 hours and then cocultured with autologous $\mathrm{T}$ cell clones. IFN- $\gamma$ secretion of $\mathrm{CD} 4^{+}$and $\mathrm{CD} 8^{+} \mathrm{T}$ cells was very low when cocultured with untargeted PBMCs. An EBNA1-Ab fusion protein targeted to langerin (CD207), which is not expressed on PBMCs, slightly induced IFN- $\gamma$ production, suggesting that alternative antigen uptake mechanisms may contribute to the background activation of $\mathrm{T}$ cells in this experimental setting. Targeting of DEC205 and CD40 significantly enhanced $\mathrm{CD} 4^{+} \mathrm{T}$ cell activation to approximately $60 \%$ of the signal obtained from peptide-pulsed PBMCs that served as a positive control (Figure 1D). Antigen delivery through DEC2O5 also yielded one of the highest responses in $\mathrm{CD} 8^{+} \mathrm{T}$ cells, and only BDCA3 targeting exceeded this and led to significant $\mathrm{CD} 8^{+} \mathrm{T}$ cell activation, with secreted IFN- $\gamma$ levels that were approximately $8 \%$ of those in the positive control (Figure 1E). Therefore, we identified BDCA3 targeting as the strongest receptor-targeting strategy for cross-presentation on MHC class I molecules. However, antigen targeting to BDCA3 did not significantly enhance cross-presentation in comparison with DEC205-directed antigen delivery.

Viral vectors have been shown to induce higher $\mathrm{CD} 8^{+} \mathrm{T}$ cell activation, therefore, we complemented our panel of EBNA1$\mathrm{Ab}$ fusion proteins with viral vectors encoding for EBNA1 or invariant chain EBNA1, namely MVAs (MVA-E1 and MVAIiE1), lentiviruses (Lenti-E1 and Lenti-IiE1), and an adenovirus 5 (Adeno-E1-LMP). PBMCs were incubated with MVAs and adenoviruses for 24 hours before coculturing with $\mathrm{T}$ cell clones and with lentiviruses for 96 hours, given their slower infection kinetics. First, we assessed EBNA1-specific CD $4^{+} \mathrm{T}$ cell activation and found that all tested viral vectors triggered a response. Notably, the addition of the invariant chain to EBNA1 in MVA-IiE1 elicited higher IFN- $\gamma$ production. Moreover, we assessed the responses of another $\mathrm{CD} 4^{+} \mathrm{T}$ cell clone specific for the AEG peptide and detected strikingly high activation levels after coculture with Adeno-E1-LMP-infected PBMCs, which reached approximately $400 \%$ of the peptide-pulsed positive control (Figure 1F). CD8 ${ }^{+} \mathrm{T}$ cell activation by Adeno-E1-LMP was as strong as the peptide-loaded positive control. Surprisingly, the MVA-IiE1 not only led to higher $\mathrm{CD} 4^{+} \mathrm{T}$ cell activation but to $\mathrm{CD}^{+} \mathrm{T}$ cell activation as well, suggesting that the 

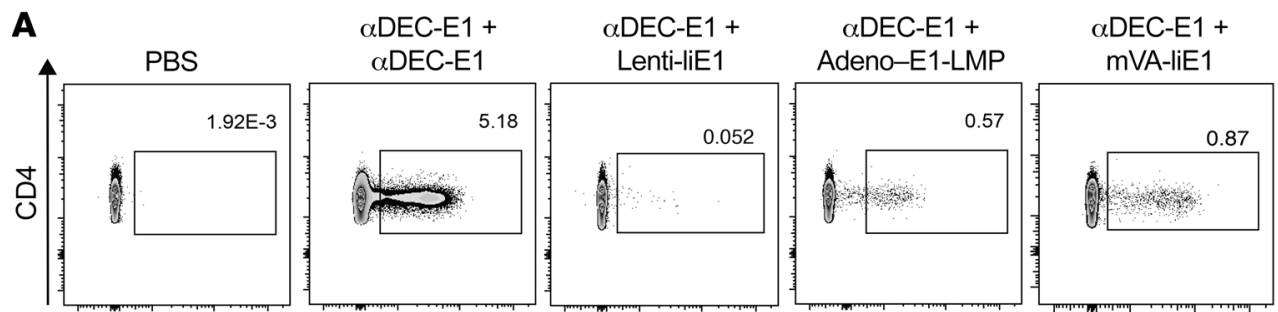

Adeno-E1-LMP +
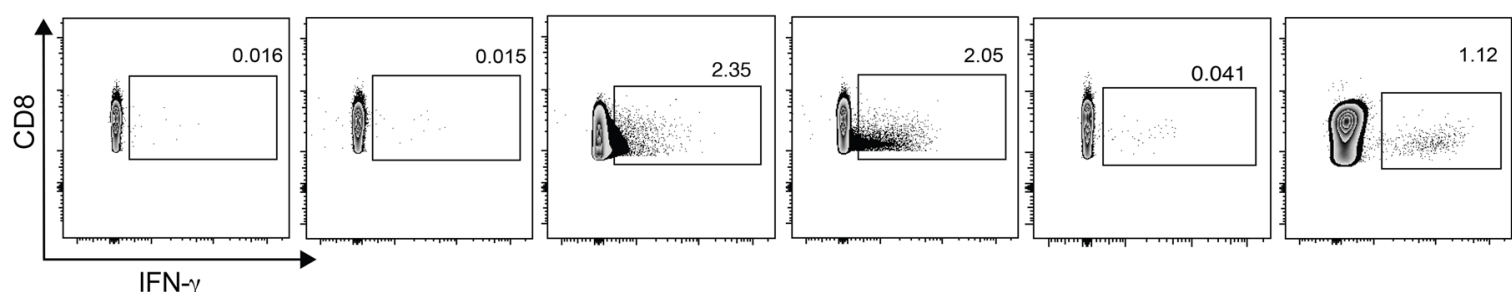

B - HCMV peptide pool

C mVA-liE1
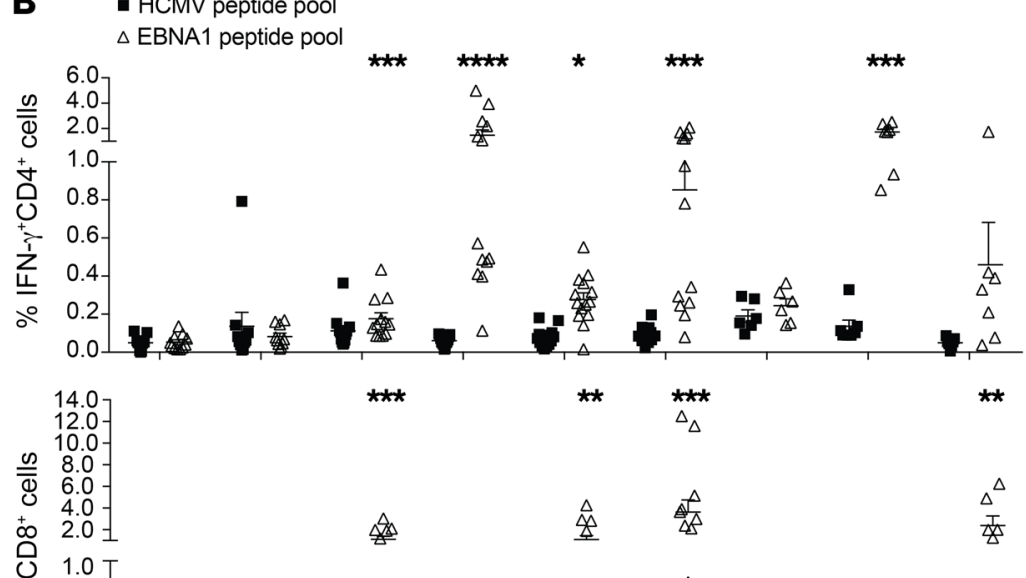

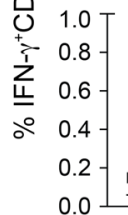
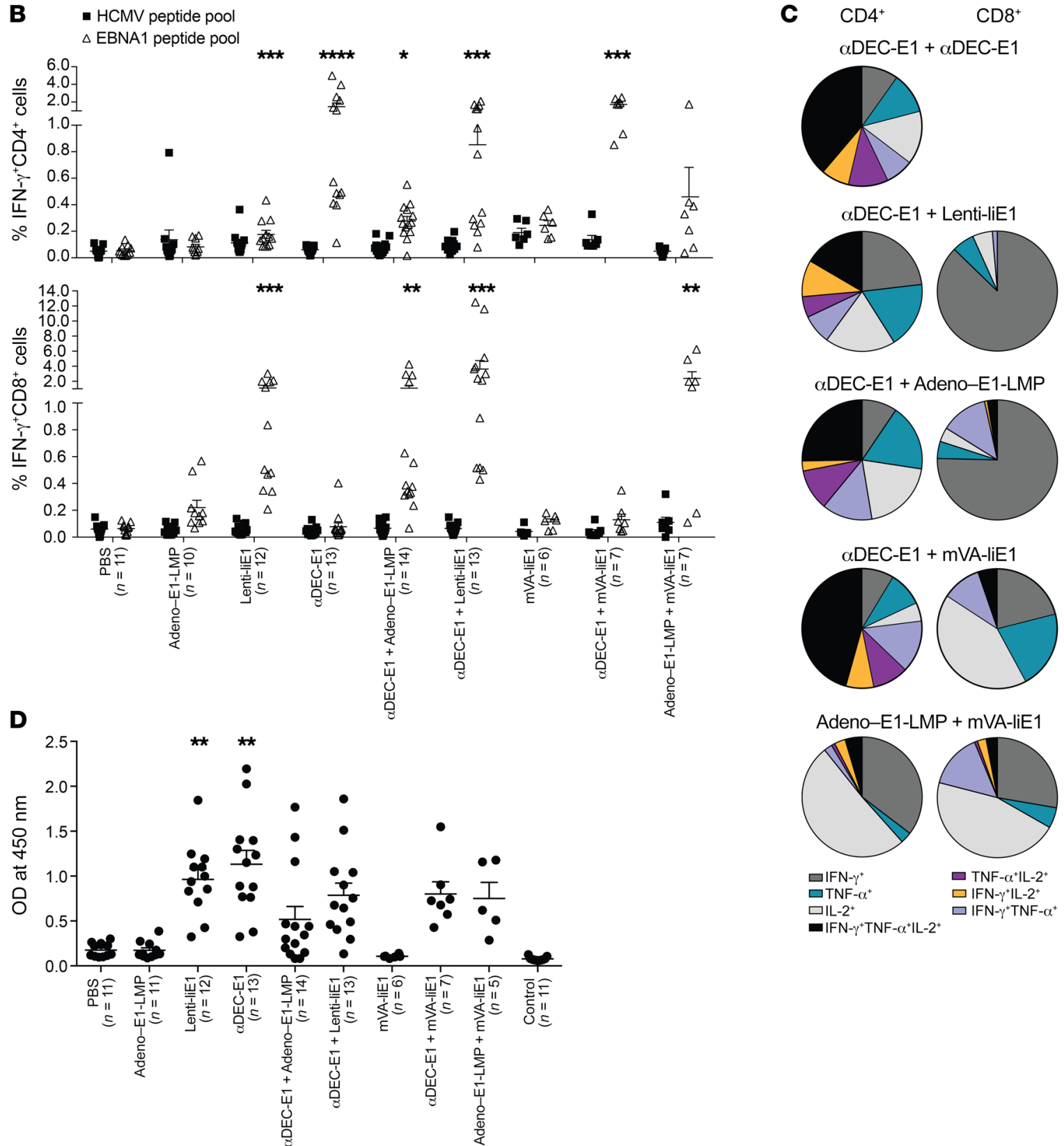

Adeno-E1-LMP + mVA-liE1

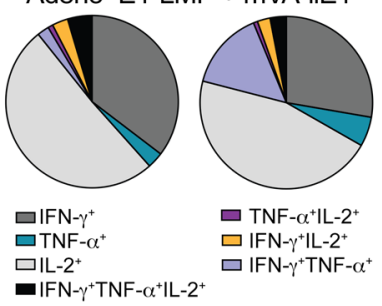


Figure 2. Comprehensive priming of mouse $\mathrm{CD4}^{+}$and $\mathrm{CD8}^{+} \mathrm{T}$ cell responses against EBNA1 by heterologous vaccination in huDEC205-Tg mice. huDEC205-Tg mice were immunized with different combinations of vaccines for the prime and the boost, which were scheduled 4 weeks apart. Mice were sacrificed 2 weeks after the boost. Bulk splenocytes were harvested and stimulated either with $1 \mu \mathrm{g} / \mathrm{ml}$ EBNA1 or control HCMV pp65 peptide pools. (A) Representative dot plots of ICS of restimulated splenocytes, gated for CD4 or CD8 expression and IFN- $\gamma$. One dot plot is shown for the PBS-treated and vaccination groups $\alpha$ DEC-E1 plus $\alpha$ DEC-E1, $\alpha$ DEC-E1 plus Lenti-liE1, $\alpha$ DEC-E1 plus Adeno-E1-LMP, $\alpha$ DEC-E1 plus MVAliE1, and Adeno-E1-LMP plus MVA-liE1 as representative examples for the data summarized in B. (B) Frequency of IFN $-\gamma^{+} \mathrm{CD} 4^{+}$and IFN- $\gamma^{+} \mathrm{CD} 8^{+}$cells from total splenocytes. Data are shown as the mean \pm SEM from 4 independent experiments with at least 3 mice per group. ${ }^{*} P<0.05$, ${ }^{* *} P<0.01$, ${ }^{* * *} P<0.001$, and ${ }^{* * * *} P<0.0001$ versus PBS-treated mice; Kruskal-Wallis test with Dunn's multiple comparison post test. (C) Cytokine profile of total splenic $C D 4^{+}$or $C D 8^{+} T$ cells in mice vaccinated with $\alpha \mathrm{DEC}-\mathrm{E} 1$ plus $\alpha$ DEC-E1, $\alpha$ DEC-E1 plus Lenti-liE1, $\alpha$ DEC-E1 plus Adeno-E1-LMP, $\alpha$ DEC-E1 plus MVA-liE1, or Adeno-E1-LMP plus MVA-liE1. Pie charts show the mean of percentage of each cytokine-secreting subset. (D) Serum obtained from mice from prime-boost experiments was analyzed for $\alpha$-EBNA1 IgC by ELISA. Each data point represents 1 individually analyzed mouse. A negative control that contained no serum was included. ${ }^{*} P<0.01$, versus PBS-treated mice; Kruskal-Wallis test with Dunn's multiple comparisons post test. Error bars indicate the SEM.

MHC class I presentation of EBNA1 benefits from the invariant chain fusion construct. Even after 96 hours of incubation, the tested lentiviruses did not induce an EBNA1-specific $\mathrm{CD}^{+} \mathrm{T}$ cell response (Figure $1 G$ ). Thus, adenoviral delivery of EBNA1 allowed for 10-fold higher $\mathrm{CD}^{+} \mathrm{T}$ cell stimulation than did any receptor targeting of EBNA1, and both MVA and adenoviruses stimulated EBNA1-specific $\mathrm{CD} 4^{+} \mathrm{T}$ cells, similar to what was observed with receptor targeting by fusion Abs.

We also performed Western blotting to analyze EBNA1 expression in virus-infected cells. The infection of HEK293T cells by MVA-E1, Lenti-E1, and Lenti-IiE1 yielded high expression of EBNA1, whereas the EBNA1 signal after MVA-IiE1 and AdenoE1-LMP infection was very low (Figure $1 C$ ). Since the constructs varied, the EBNA1 band was visible at different molecular weights. MVA-E1 carries EBNA1 without the Gly/Ala repeat and runs at approximately $45 \mathrm{kDa}(25)$, and MVA-IiE1 migrates more slowly (at a higher molecular weight) because of the additional invariant chain protein. Lenti-E1 carries only the most immunogenic part of EBNA1, the C-terminus from aa 400-641, with an approximate size of $30 \mathrm{kDa}$. Infection with Adeno-E1-LMP also leads to expression of the Gly/Ala repeat-deleted EBNA1 protein, however, with additional LMP polyepitopes (26), it migrates at approximately $60 \mathrm{kDa}$. The analysis of virus-infected PBMCs showed a slightly different trend: MVA-IiE1 and MVA-E1 led to strong EBNA1 expression, whereas even after 96 hours of infection, Lenti-IiE1 only yielded low expression of EBNA1 (Supplemental Figure 1, C and D). The lower-molecular-weight bands, seen after MVA-IiE1 and MVA-E1 infection of PBMCs, were possibly degraded EBNA1 protein. The high activation of $\mathrm{T}$ cell clones after coculture with Adeno-E1-LMP-infected PBMCs could not be directly correlated with high EBNA1 expression in infected cells.

Comprehensive priming of mouse $C D 4^{+}$and $C D 8^{+} T$ cell responses against EBNA1 by heterologous vaccination. To investigate the different capacities of receptor-targeting strategies and viral vector infections to induce EBNA1-specific T cell responses, we developed homologous and heterologous prime-boost vaccinations in a human DEC205-Tg (huDEC205-Tg) C57BL/6 mouse model. We focused on DEC2O5 targeting, as it elicited one of the highest $\mathrm{CD}^{+}$and $\mathrm{CD}^{+}{ }^{+} \mathrm{T}$ cell responses in our in vitro experiments, and because targeting to other receptors did not result in substantially improved cross-presentation of EBNA1 on MHC class I molecules for $\mathrm{CD}^{+} \mathrm{T}$ cell stimulation. We combined DEC205 targeting of antigen with the most promising viral vectors, namely, AdenoE1-LMP and MVA-IiE1, as well as Lenti-IiE1, given that lentiviral vectors have been extensively explored in virus-based therapies (reviewed in ref. 27). In both the heterologous and homologous vaccine regimens, boosting vaccines were injected 4 weeks after the priming vaccines. In comparison, administration of AdenoE1-LMP prime plus MVA-IiE1 boost was recently introduced as a promising vaccination approach for malaria (28). $\mathrm{CD} 4^{+}$and $\mathrm{CD} 8^{+}$ $\mathrm{T}$ cell responses toward the EBNA1 antigen were analyzed using intracellular cytokine staining (ICS) of IFN- $\gamma$ after restimulation of splenic cells for 5 hours with an EBNA1 peptide library that covered aa 400-641 (Figure 2A and Supplemental Figure 2A). The highest $\mathrm{CD}^{+} \mathrm{T}$ cell response was induced by the homologous immunization with $\alpha \mathrm{DEC}-\mathrm{E} 1$ and poly(I:C) as an adjuvant (Figure 2B). Adeno-E1-LMP, Lenti-IiE1, and MVA-IiE1 only elicited mild or no $\mathrm{CD}^{+} \mathrm{T}$ cell responses in vivo, which improved markedly when these viral vectors were preceded by $\alpha$ DEC-E1. We also observed an increase in the frequency of IFN- $\gamma$-secreting $\mathrm{CD} 4^{+} \mathrm{T}$ cells after heterologous prime-boost with Adeno-E1-LMP and MVA-IiE1 compared with either vector alone. However, the effect of vaccine combinations on the $\mathrm{CD} 8^{+} \mathrm{T}$ cell compartment was much more striking. We detected a strong and significant increase in $\mathrm{CD}^{+} \mathrm{T}$ cell responses after vaccination with $\alpha$ DEC-E1 followed by adenoviral or lentiviral vectors, with only Lenti-IiE1 being able to prime EBNA1-specific CD8 ${ }^{+} \mathrm{T}$ cells on its own. MVA-IiE1 alone or in combination with receptor targeting did not induce IFN- $\gamma$-secreting $\mathrm{CD}^{+} \mathrm{T}$ cells, whose frequency was significantly enhanced, however, after priming with AdenoE1-LMP (Figure 2B). Hence, it can be concluded that heterologous prime-boost vaccination increases the level of EBNA1-specific $\mathrm{CD}^{+} \mathrm{T}$ cell responses in huDEC205-Tg mice in comparison with homologous prime-boost vaccination. Of note, using IFN- $\gamma$ ELISPOT and restimulation of splenic cells after vaccination with all single peptides of the EBNA1 peptide mix, we showed that the EBNA1-specific $T$ cell responses were distributed quite evenly over the whole length of the EBNA1 protein, with stronger peptide recognition in 4 clusters (Supplemental Figure 2B). In addition to IFN- $\gamma$, other Th1 cytokines such as TNF- $\alpha$ and IL-2 have been shown to play a role in antiviral and/or antitumor immunity $(29,30)$. To assess the number of polyfunctional EBNA1-specific $\mathrm{T}$ cells after different vaccination schemes, we analyzed their cytokine expression profile by ICS. In general, CD4 ${ }^{+} \mathrm{T}$ cells showed a more pronounced polyfunctional phenotype than did $\mathrm{CD}^{+} \mathrm{T}$ cells. DEC205 targeting led to the highest percentage of $\mathrm{CD}^{+} \mathrm{T}$ cells that produced either 2 or 3 of the above-mentioned cytokines, irrespective of the vaccination strategy (Figure 2C). Viral vectors induced a high number of $\mathrm{CD} 4^{+} \mathrm{T}$ cells that produced IFN- $\gamma$, TNF- $\alpha$, or IL-2 alone (Supplemental Figure 2C). Generally, $\mathrm{CD}^{+} \mathrm{T}$ cells followed the same trend. After Adeno- 

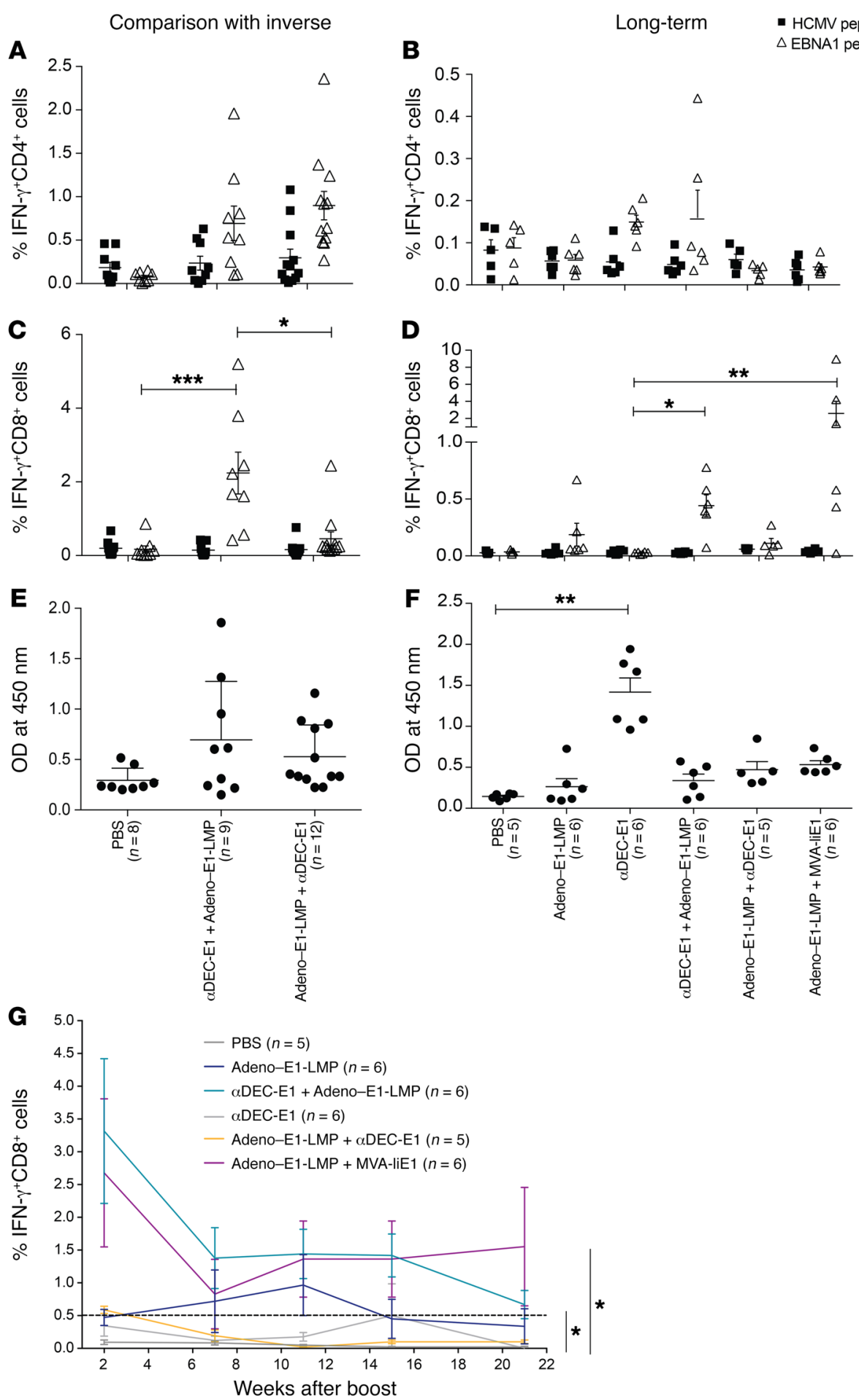

E1-LMP vaccination, we observed polyfunctionality, which was slightly decreased after combination with $\alpha$ DEC-E1 priming. Also, $\alpha$ DEC-E1 plus MVA-IiE1 vaccination led to polyfunctional $\mathrm{CD}^{+} \mathrm{T}$ cell responses, which was not observed with homologous MVA-IiE1 vaccination alone. Interestingly, MVA-IiE1 vaccination led to the highest number of IL-2-secreting $\mathrm{CD}^{+} \mathrm{T}$ cells, independent of combination with $\alpha$ DEC-E1 or Adeno-E1-LMP. Most successful vaccines induce not only robust $\mathrm{T}$ cell responses but also functional $\mathrm{Ab}$ responses. Therefore, we investigated whether the different homologous and heterologous vaccination schemes lead to $\alpha$ EBNA1 IgG Ab titers in the serum of vaccinated animals. In line with the strong and diverse $\mathrm{CD} 4^{+} \mathrm{T}$ cell responses, the homologous prime-boost regimen with $\alpha \mathrm{DEC}-\mathrm{E} 1$ led to the highest $\alpha$ EBNA1 IgG Ab titers (Figure 2D). Among the investigated viral vectors studied, only Lenti-IiE1 led to high $\alpha$ EBNA1 IgG Ab titers, which correlated with the $\mathrm{CD}^{+} \mathrm{T}$ cell responses that were induced by Lenti-IiE1. As soon as $\alpha$ DEC-E1 was applied as a priming vaccine, $\alpha$ EBNA1 IgG Ab titers were also found to be elevated 
A

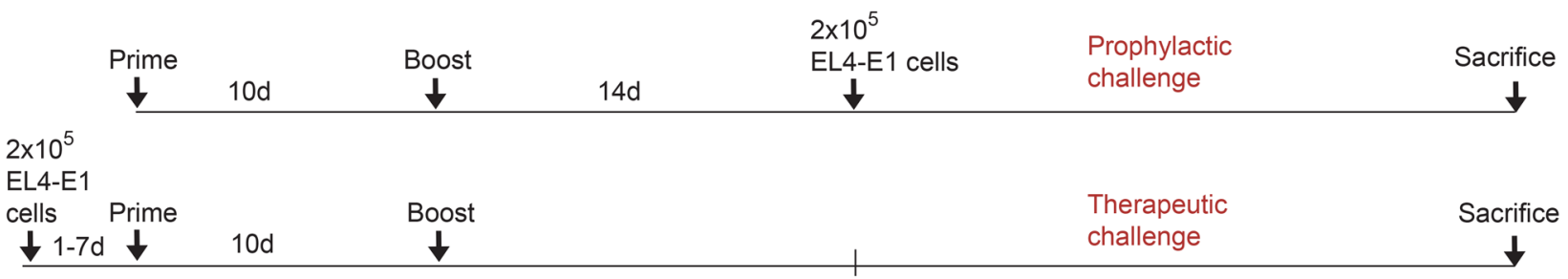

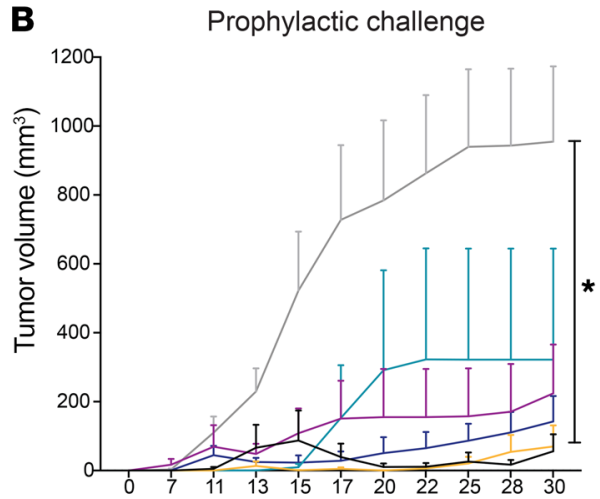

Days after tumor cell injection
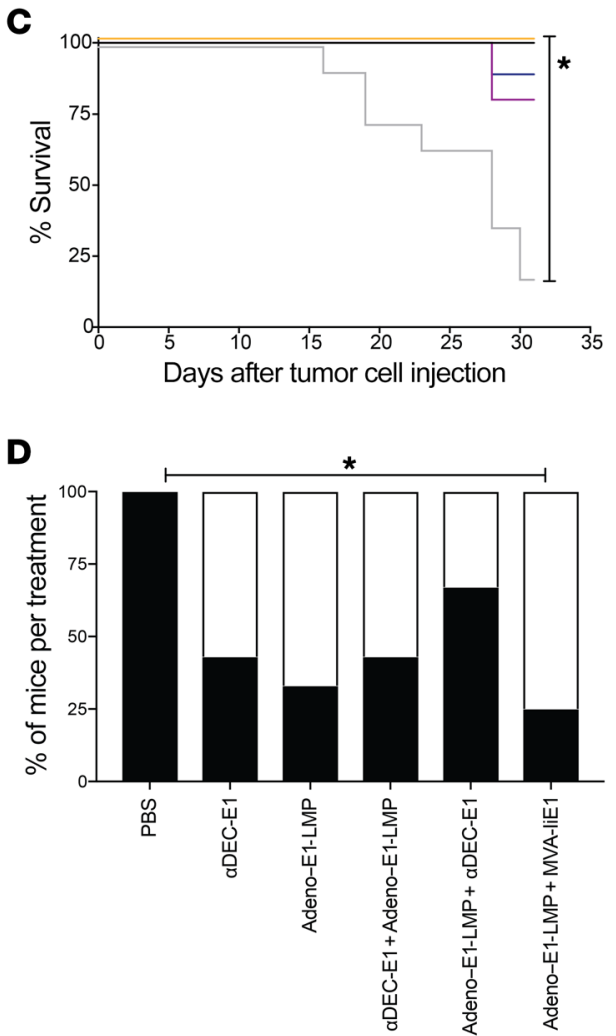

E

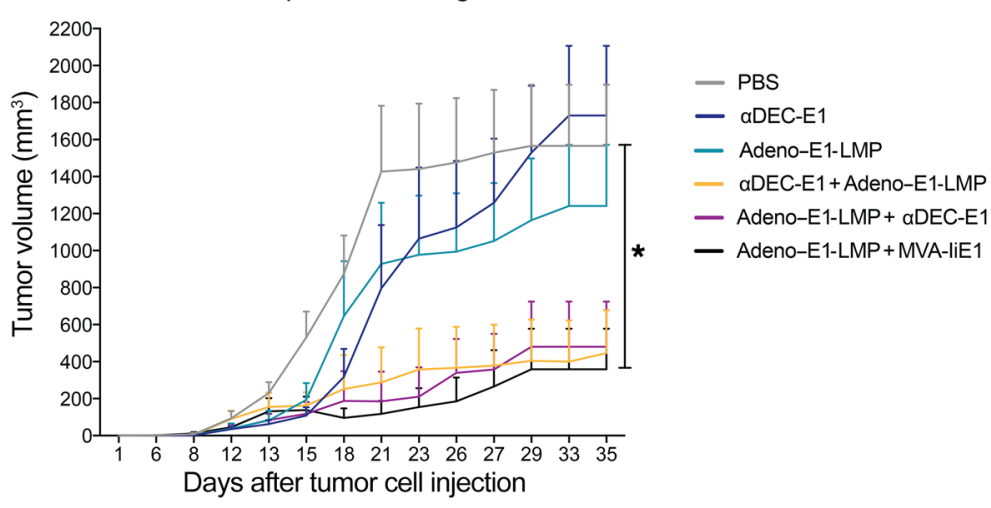

$\mathbf{F}$
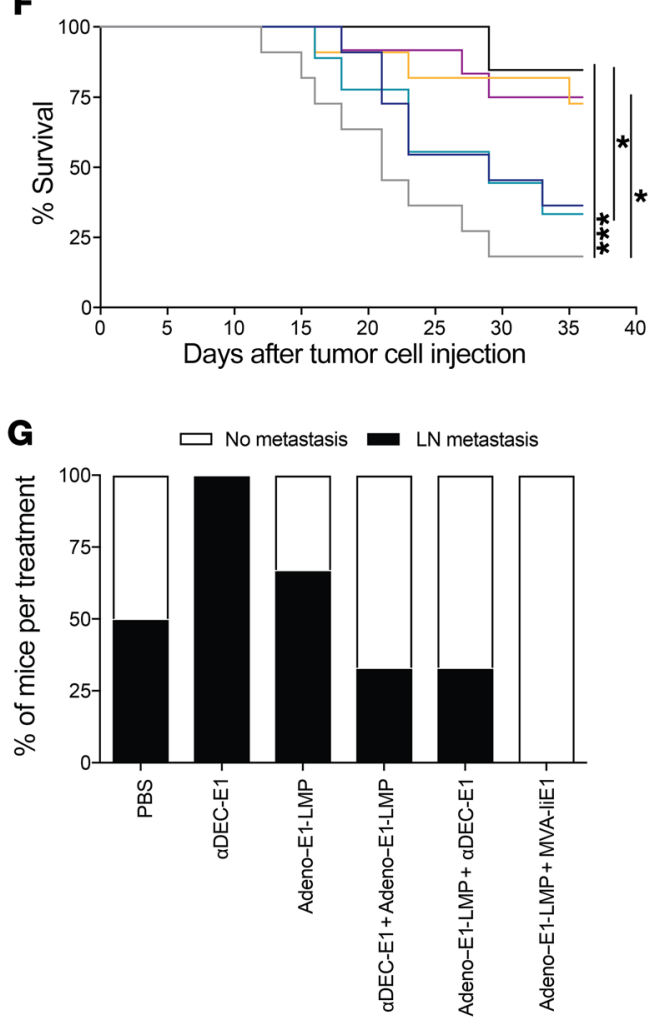

Figure 4. Protection from EBNA1-expressing EL4 lymphoma challenge by heterologous prime-boost vaccination in huDEC205-Tg mice. (A) hHuDEC205-Tg mice were immunized with different combinations of vaccines for the prime and the boost, which were scheduled 10 days apart. Mice were s.c. challenged with $2 \times 10^{5}$ EBNA1-expressing EL4 cells (EL4-E1) either 14 days after the boost in a prophylactic setting (B, C, and $\mathbf{D}$ ) or 1 to 7 days before the prime vaccination in a therapeutic setting (E, F, and $\mathbf{G})$. Mice were monitored every second day, weight was measured, and tumor size was analyzed by caliper. Mice were sacrificed when the tumor reached $15 \mathrm{~mm}$ or more in diameter. (B and $\mathbf{E}$ ) The tumor volume was calculated using the formula $A^{2} \times B \times 0.52$, where $A$ is the shortest diameter perpendicular to the longest diameter, and B is the longest diameter. The mean tumor volume plus the SD of 3 independent experiments with at least 3 mice per group is shown. ${ }^{*} P<0.05$ versus PBS-treated mice; 2 -way ANOVA with Tukey's multiple comparisons test. (C and $\mathbf{F}$ ) The percentage survival from 3 independent experiments with at least 3 mice per group is shown. ${ }^{*} P<0.05$ and ${ }^{* * *} P<0.001$; Mantel-Cox test. ( $\mathbf{D}$ and $\mathbf{G}$ ) At sacrifice, bulk single-cell suspensions of $L N$ cells were harvested and analyzed by EBNA1 qPCR from representative prophylactic (D) and therapeutic (G) EL4-E1 tumor challenges. Abundance of the EBNA1 gene was normalized to the $U B C$ gene as the tumor load. A tumor-load cutoff of 0.005 or greater was set. The percentage of mice per condition with and without tumor burden in the LNs is depicted. Statistical analysis was done by 2-way ANOVA with Tukey's multiple comparisons test using the quantitation cycle $\left(c_{q}\right)$ value of the qPCR. 
along with the other viral vectors Adeno-E1-LMP and MVA-IiE1. Surprisingly, the combination of Adeno-E1-LMP and MVA-IiE1 also induced good $\mathrm{Ab}$ responses toward EBNA1. However, these $\mathrm{Ab}$ responses against the nuclear EBNA1 antigen probably do not contribute to protection but rather indicate the magnitude of the corresponding $\mathrm{CD}^{+} \mathrm{T}$ cell responses. These studies indicated that $\alpha \mathrm{DEC}-\mathrm{E} 1$ plus Adeno-E1-LMP1, $\alpha \mathrm{DEC}-\mathrm{E} 1$ plus Lenti-IiE1, and Adeno-E1-LMP plus MVA-IiE1 elicit the highest balanced $\mathrm{CD}^{+}$and $\mathrm{CD}^{+} \mathrm{T}$ cell responses against EBNA1. Lentiviruses can possibly cause harm in the host because of gene dysregulation that can occur after lentiviral genome insertion into the host genome. Because $\alpha$ DEC-E1 plus Lenti-IiE1 did not confer a clear advantage compared with $\alpha$ DEC-E1 plus Adeno-E1-LMP1 vaccination, we focused our vaccination strategies on Adeno-E1-LMP1.

Persistent and potent EBNA1-specific $C D 8^{+} T$ cell responses upon comprehensive $C D 4^{+}$and $C D 8^{+} T$ cell priming by heterologous vaccination. It has been shown that $\mathrm{CD} 4^{+} \mathrm{T}$ cell help is not only needed for $\mathrm{CD} 8^{+} \mathrm{T}$ cell priming but also for maintaining protective $\mathrm{CD} 8^{+} \mathrm{T}$ cell memory. $\mathrm{CD}^{+} \mathrm{T}$ cells have been shown to assist in the priming of protective $\mathrm{CD}^{+} \mathrm{T}$ cell responses by CD40L- and CD40-mediated DC maturation (31-33) and to maintain $\mathrm{CD}^{+} \mathrm{T}$ cell function via IL-2 and IL-21 $(34,35)$. Therefore, antigen-specific $\mathrm{CD}^{+} \mathrm{T}$ cell responses might augment priming of $\mathrm{CD}^{+} \mathrm{T}$ cells against the same antigen. In order to investigate the effect of $\mathrm{CD} 4^{+} \mathrm{T}$ cell help by $\alpha \mathrm{DEC}-\mathrm{E} 1$ priming before vaccination with viral vectors, the heterologous prime-boost immunization schemes were inversed. In the inverse heterologous prime-boost vaccination, the viral vector Adeno-E1-LMP was used as a priming vaccine and $\alpha$ DEC-E1 as a boost. Comparison of standard versus inverse heterologous prime-boost regimens showed that priming with $\alpha$ DEC-E1 augmented $\mathrm{CD}^{+} \mathrm{T}$ cell priming induced by Adeno-E1-LMP (Figure 3B). The inversion led to significantly lower EBNA1-specific CD8 ${ }^{+}$ $\mathrm{T}$ cell responses. In contrast, $\mathrm{CD} 4^{+} \mathrm{T}$ cell responses as well as the amount of $\alpha$ EBNA1 IgG in the serum of vaccinated mice were not affected by the inversion (Figure 3, A and C). The sole effect on $\mathrm{CD}^{+} \mathrm{T}$ cell responses supports our hypothesis that the $\mathrm{CD} 8^{+} \mathrm{T}$ cell priming and maintenance during our heterologous prime-boost vaccination is dependent on $\mathrm{CD} 4^{+} \mathrm{T}$ cell help.

To assess how long the vaccinated mice were capable of eliciting $\mathrm{T}$ cell responses to EBNA1, the mice were observed until week 21 after the boost. PBMCs were collected 2 weeks after the boost and then approximately once a month to test for restimulation capacity after an EBNA1 peptide pulse in ICS (Figure 3D). We found that $\mathrm{CD} 4^{+} \mathrm{T}$ cell responses were generally low in the periphery but were most prominent after $\alpha$ DEC-E1 vaccination (data not shown). CD8 ${ }^{+} \mathrm{T}$ cell responses peaked in week 2 after the boost. The heterologous prime-boost vaccines Adeno-E1-LMP plus MVA-IiE1 and $\alpha$ DEC-E1 plus Adeno-E1-LMP induced the highest EBNA1-specific CD8 ${ }^{+} \mathrm{T}$ cell responses in the blood, with the latter remaining consistently high over a 15 -week period. This long-term immunity was not seen after $\alpha$ DEC-E1 plus Lenti-IiE1, which may be partly due to the lower ability of the Lenti-IiE1 boosting vaccine to induce $\mathrm{CD}^{+} \mathrm{T}$ cell help (data not shown). To assess the long-term EBNA1-specific immune responses in greater detail, all mice were sacrificed 21 weeks after the boost. Splenic cells were restimulated with an EBNA1 peptide library and a human cytomegalovirus pp65 (HCMV pp65) peptide library as a negative control (Figure 3, E and F). The overall $\mathrm{CD}^{+}$as well as $\mathrm{CD}^{+} \mathrm{T}$ cell responses were lower than those in the short-term experiments, but EBNA1-specific CD4 ${ }^{+} \mathrm{T}$ cell responses were still detectable at the time of sacrifice but at similar levels across all immunization groups (Figure 3E). After $\alpha \mathrm{DEC}-\mathrm{E} 1$ plus AdenoE1-LMP immunization, we detected higher percentages of EBNA1-specific $\mathrm{CD} 4^{+} \mathrm{T}$ cells in comparison with percentages seen with inverse Adeno-E1-LMP plus $\alpha$ DEC-E1 and Adeno-E1-LMP plus MVA-IiE1 vaccination. Mice in the heterologous prime-boost vaccination regimens showed slightly higher antigen-specific $\mathrm{CD}^{+} \mathrm{T}$ cell responses than did mice given viral vector vaccines alone (Figure 3F). Interestingly, even 21 weeks after the boost, the deficit in EBNA1-specific $\mathrm{CD}^{+} \mathrm{T}$ cell responses, comparing $\alpha$ DEC-E1 plus Adeno-E1-LMP with inverse prime-boost AdenoE1-LMP plus $\alpha$ DEC-E1, was significant. We also assessed B cell responses toward EBNA1 at this time point and found significantly higher $\alpha$ EBNA1 Ab titers only in mice vaccinated with $\alpha$ DEC-E1 compared with titers detected in the nonvaccinated (PBS-treated) mice (Figure 3G). $\alpha$ DEC-E1 plus Adeno-E1-LMP and AdenoE1-LMP plus MVA-IiE1 regimens mostly produced similar results, except with respect to the longevity of the $\mathrm{CD} 8^{+} \mathrm{T}$ cell response. This $\mathrm{CD}^{+} \mathrm{T}$ cell lifespan was further increased in animals vaccinated with Adeno-E1-LMP plus MVA-IiE1 compared with animals vaccinated with $\alpha$ DEC-E1 plus Adeno-E1-LMP, with the latter group having a more diverse cytokine profile within the $\mathrm{CD} 8^{+} \mathrm{T}$ cell population. Hence, we chose $\alpha$ DEC-E1 plus Adeno-E1-LMP and Adeno-E1-LMP plus MVA-IiE1 for further studies.

Protection from EBNA1-expressing EL4 lymphoma challenge by heterologous vaccination. To evaluate the therapeutic effect of the most potent heterologous prime-boost vaccinations, namely, $\alpha$ DEC-E1 plus Adeno-E1-LMP and Adeno-E1-LMP plus MVA-IiE1, we developed an EBNA1 ${ }^{+}$model tumor. EL4, a T cell lymphoma cell line, was infected with Lenti-EBNA1-GFP. GFP ${ }^{+}$ lymphoma cells were enriched by FACS and assessed for EBNA1 expression by Western blotting and IHC (Supplemental Figure 3, A and D). We performed histological analysis of untreated EL4-E1 tumors and observed positive staining for CD4 and FoxP3. Only a few tumor-infiltrating $\mathrm{CD}^{+} \mathrm{T}$ cells were detected (Supplemental Figure 3B). Mice were vaccinated with either $\alpha$ DEC-E1 plus Adeno-E1-LMP or Adeno-E1-LMP plus MVA-IiE1 or homologously with $\alpha \mathrm{DEC}-\mathrm{E} 1$ or Adeno-E1-LMP as a comparison. Also, 1 inverse prime-boost vaccination, Adeno-E1-LMP plus $\alpha$ DEC-E1, was included to assess the importance of the order of vaccines in prime-boost regimens. Mice were challenged with EL4-E1 tumor cells on 2 different schedules and then given either prophylactic or therapeutic vaccination (Figure $4 \mathrm{~A}$ ). In the prophylactic vaccination group, 2 weeks after the boost, mice were injected s.c. with $2 \times 10^{5}$ EL4-E1 cells/mouse. In the therapeutic vaccination group, mice were challenged on day 0 , and immunization followed within 1 to 7 days. Tumor burden was analyzed every second day by caliper measurement. In the challenge after $\alpha$ DEC-E1 plus AdenoE1-LMP or Adeno-E1-LMP plus MVA-IiE1 prophylactic vaccination, 11 of 13 mice showed complete EL4-E1 tumor rejection (Figure 4B). The survival rate of these mice was increased from $10 \%$ to $100 \%$ (Figure $4 \mathrm{C}$ ). Homologous vaccinations led to slower and decreased tumor growth, which was comparable to the tumor growth observed in the inverse prime-boost group. The spread 
A

\begin{tabular}{|c|c|c|c|c|c|c|}
\hline $\mathbf{A}$ & & & & $2 \times 10^{5}$ & & Con \\
\hline Prime & $10 \mathrm{~d}$ & Boost & $11 \mathrm{~d}$ & EL4-E1 cells & $31 \mathrm{~d}$ & Sacri \\
\hline
\end{tabular}

B

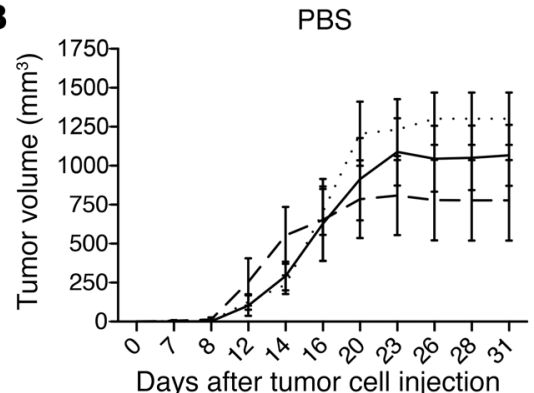

C

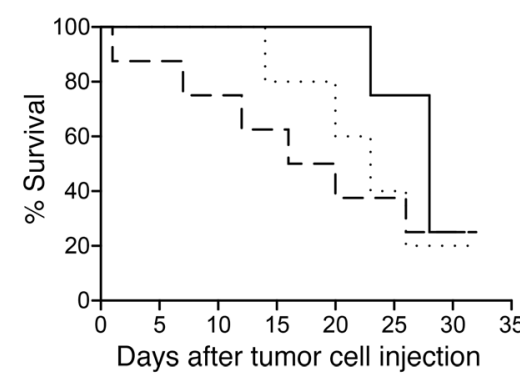

- Nondepleted

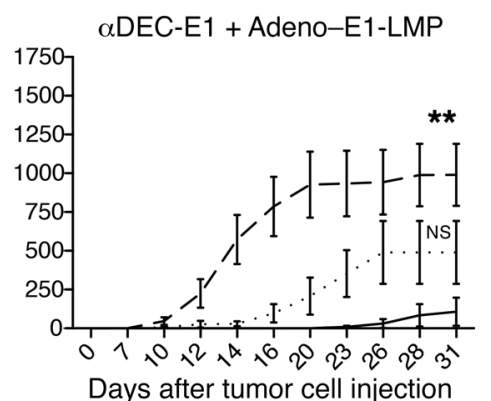

Days after tumor cell injection
Adeno-E1-LMP + MVA-liE1

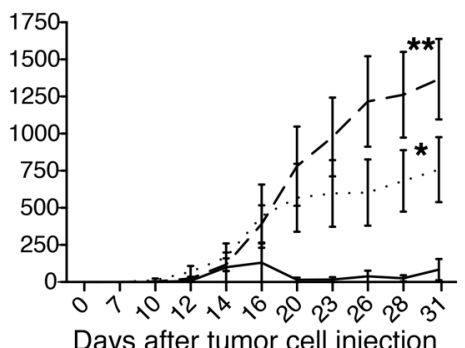

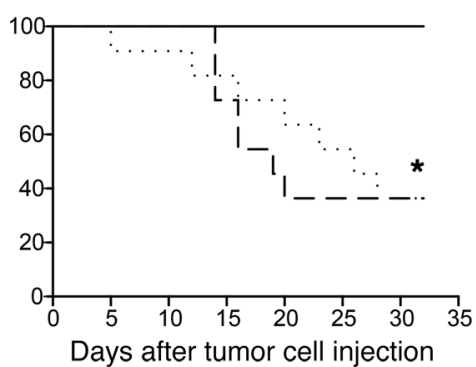

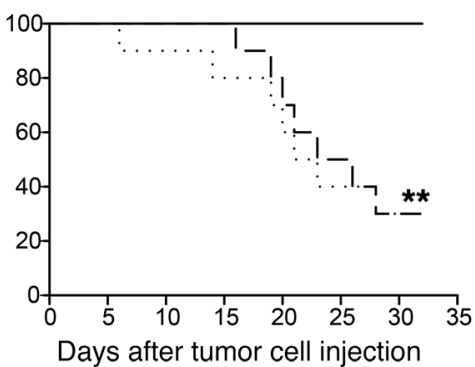

- $\mathrm{CD}^{+} \mathrm{T}$ cell depletion $\quad \cdots \mathrm{CD}^{+} \mathrm{T}$ cell depletion

D

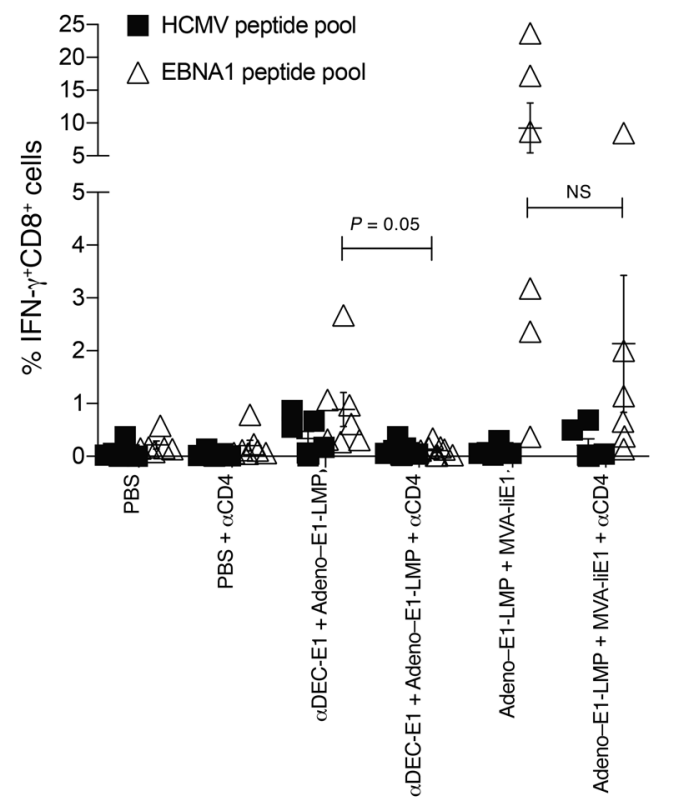

E

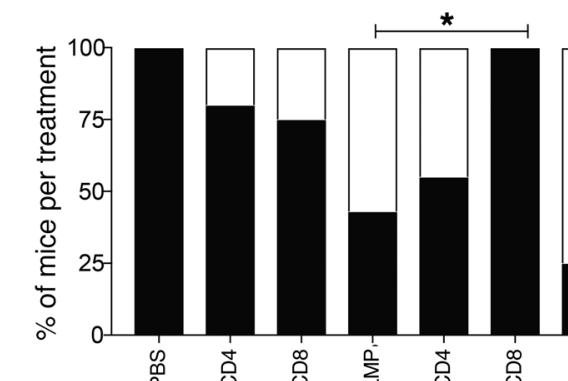

$\square$ No metastasis
LN metastasis

$\square$ No metastasis
LN metastasis

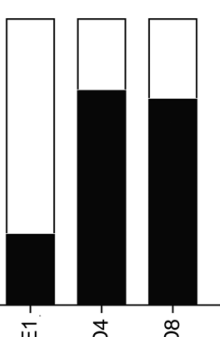

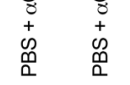

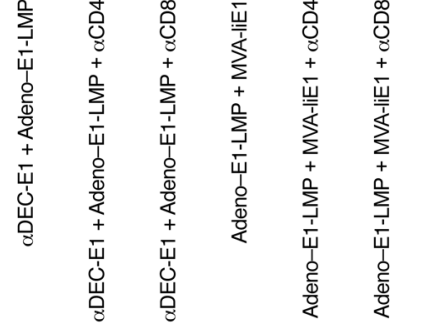

Figure 5. Dependence on CD4 ${ }^{+}$and $\mathrm{CD8}^{+} \mathrm{T}$ cell populations for protection from EL4-E1 challenge after heterologous vaccination. (A) huDEC205-Tg mice were immunized with different combinations of vaccines for the prime and boost scheduled 10 days apart. Before the prime and before the boost, mice were depleted with injections of $\alpha \mathrm{CD} 4$ or $\alpha \mathrm{CD} 8 \mathrm{Ab}$ on 3 consecutive days. Mice were challenged with $2 \times 10^{5}$ EL4-E1 cells s.c. 14 days after the boost. Mice were monitored every second day, weight was measured, and tumor size was analyzed by caliper. (B) Tumor growth was determined every second to third day. Tumor volume was calculated by the formula: $A^{2} \times B \times 0.52$. The mean tumor volume plus the SD of the experiment with 6 mice per group is shown. ${ }^{*} P<0.05$ and ${ }^{* *} P<0.01 ; 2$-way ANOVA and Tukey's multiple comparisons test. (C) Mice were sacrificed when the tumor reached $15 \mathrm{~mm}$ or more in diameter. The percentage survival from 1 experiment with 6 mice per group is shown. ${ }^{*} P<0.05$ and ${ }^{* *} P<0.005$; Mantel-Cox test. (D) At the point of sacrifice, bulk splenocytes were harvested and stimulated either with $1 \mu \mathrm{g} / \mathrm{ml}$ EBNA1 or control HCMV pp65 peptide pool. IFN-y production was monitored by ICS in CD8 $8^{+}$gated cells. The mean \pm SEM from 1 experiment with 6 mice per group is shown. $P=0.05$; Kruskal-Wallis with Dunn's multiple comparisons post test. (E) At sacrifice, bulk single-cell suspensions of cells from LNs were harvested and analyzed by EBNA1 qPCR. Abundance of EBNA1 gene was normalized to the UBC gene. Data are shown as the mean \pm SD from experiments with 6 mice per group. ${ }^{*} P<0.05$; statistical analysis was done by 2-way ANOVA and Tukey's multiple comparisons test using the $\mathrm{c}_{\mathrm{q}}$ value of the qPCR. 
A

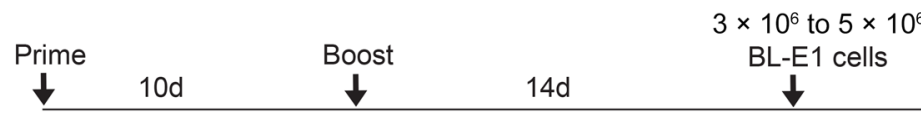
$45 d$

Sacrifice
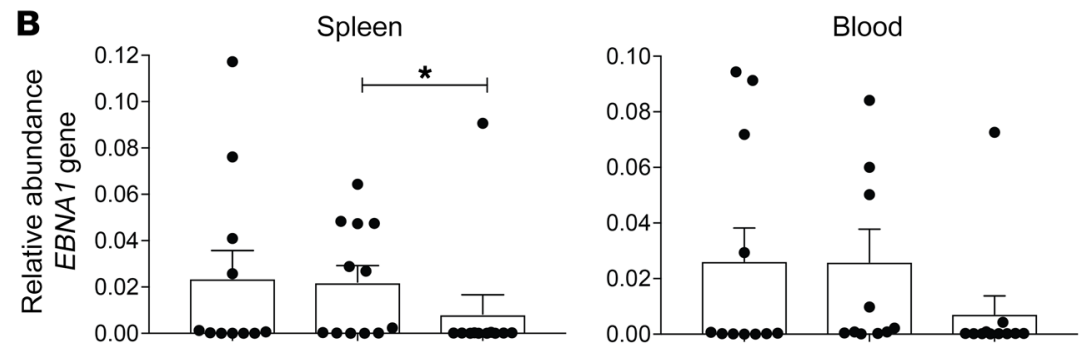

C $\square$ w/otumor
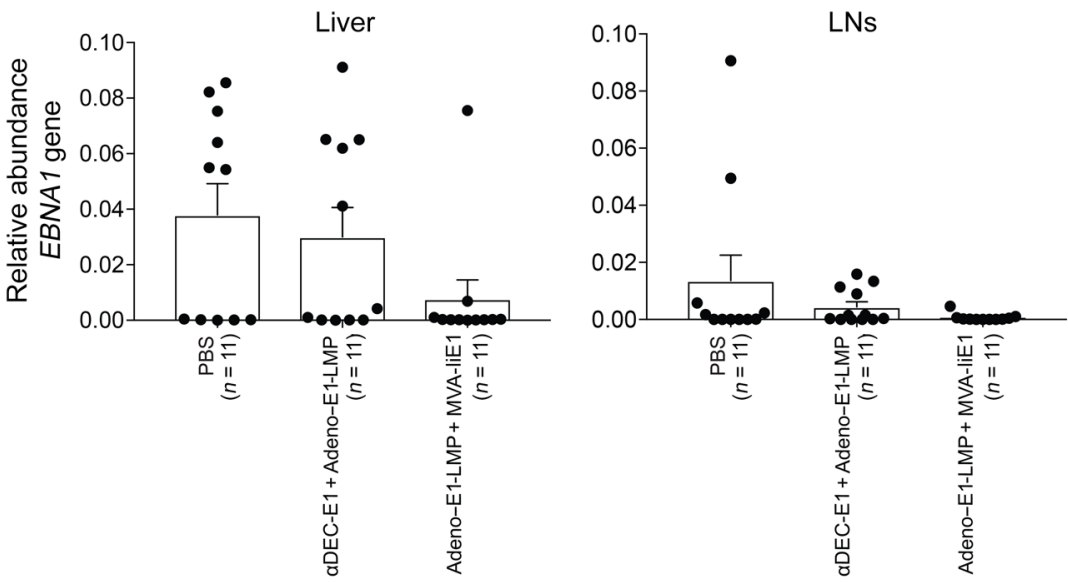

$$
\begin{aligned}
& \square \text { one organ } \\
& \square \text { two organs }
\end{aligned}
$$

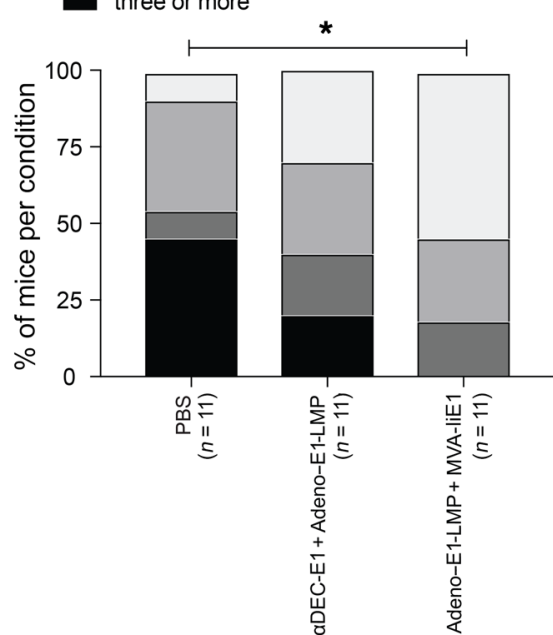

D

PBS, no tumor
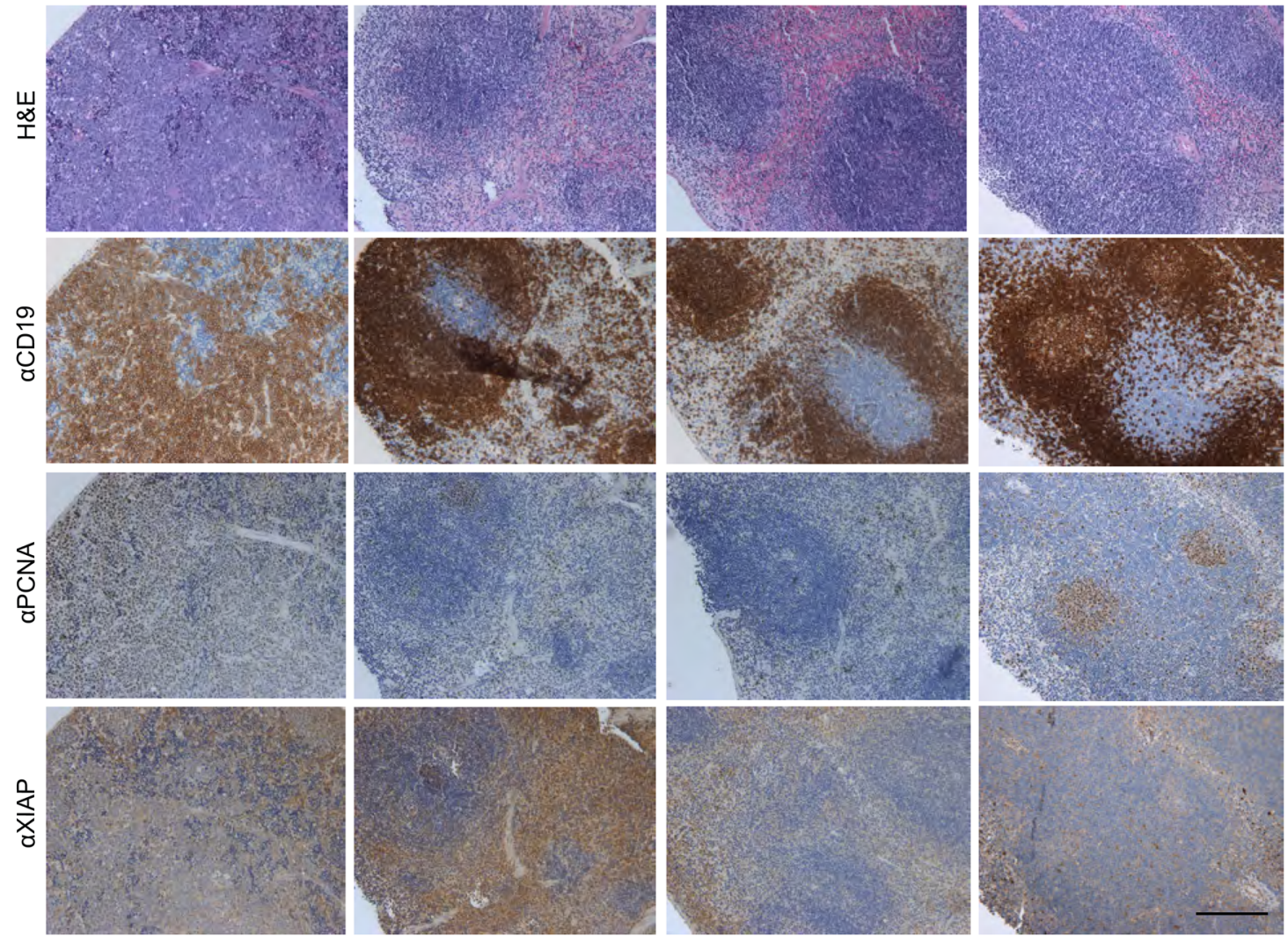
Figure 6. Protection from EBNA1-induced B cell lymphoma challenge by heterologous vaccination. (A) huDEC205-Tg mice were immunized with different combinations of vaccines for the prime and the boost, scheduled 10 days apart. Mice were challenged with $3 \times 10^{6}$ to $5 \times 10^{6}$ EBNA1+ $B$ cell tumor cells (BL-E1) i.v. 14 days after the boost in a preventive setting. Mice were monitored every second day, including measurement of weight, observation of general behavior, and assessment using the mouse grimace scale. (B) At sacrifice, bulk single-cell suspensions of cells from LNs, spleen, liver, and blood were harvested and analyzed by EBNA1 qPCR. Abundance of the EBNA1 gene was normalized to the $U B C$ gene as the tumor load. Data are shown as the mean \pm SD from 2 independent experiments with at least 5 mice per group. (C) A tumor load cutoff of 0.005 or higher was set, and results of all analyzed organs from each mouse were pooled. The percentage of mice per condition without tumor burden and with tumor burden in 1 to 4 organs is depicted. ${ }^{*} P<0.05$; Mantel-Cox test. (D) At sacrifice, splenic tissue from treated mice with EBNA1-induced B cell lymphoma were fixed in PFA and embedded in paraffin. Splenic tissues from PBS-treated mice without tumor treatment were used. Splenic tissue samples were stained with $\mathrm{H} \& \mathrm{E}, \alpha \mathrm{CD} 19, \alpha \mathrm{PCNA}$, and $\alpha \mathrm{XIAP}$ Abs. One representative staining for each group is shown. Scale bar: $20 \mu \mathrm{m}$.

of EL4-E1 tumor cells into lymph nodes (LNs) was significantly decreased only with Adeno-E1-LMP plus MVA-IiE1 vaccination (Figure 4D). In mice in the therapeutic vaccination group, EL4-E1 tumor growth was significantly decreased and slowed down with $\alpha$ DEC-E1 plus Adeno-E1-LMP and Adeno-E1-LMP plus MVA-IiE1 treatment (Figure 4E). The survival rate of heterologously vaccinated animals was increased to approximately $75 \%$ (Figure $4 \mathrm{~F}$ ). During the therapeutic challenge, we found no difference between standard and inverse $\alpha$ DEC-E1 plus Adeno-E1-LMP, which could suggest that early $\mathrm{CD}^{+} \mathrm{T}$ cell responses are of similar importance to sustained $\mathrm{CD}^{+} \mathrm{T}$ cell responses upon $\mathrm{CD} 4^{+} \mathrm{T}$ cell help. Another hypothesis to explain this phenomenon would be that the tumor may have already primed $\mathrm{T}$ cell responses, and therefore priming by DEC20 5 targeting would be less effective than in the preventive setting. Interestingly, homologous vaccinations had only a mild effect on tumor growth and survival in the therapeutic setting. However, the spread of EL4-E1 tumor cells into LNs during the therapeutic challenge was prevented only with Adeno-E1-LMP plus MVA-IiE1 vaccination (Figure $4 \mathrm{G}$ ). Thus, both $\alpha$ DEC-E1 plus Adeno-E1-LMP and Adeno-E1-LMP plus MVA-IiE1 vaccinations performed best as prophylactic or therapeutic treatments during EL4-E1 challenge.

Dependence on $\mathrm{CD}^{+}$and $\mathrm{CD} 8^{+} \mathrm{T}$ cell populations for protection from EL4-E1 challenge after heterologous vaccination. To understand the dependence of the $\alpha$ DEC-E1 plus Adeno-E1-LMP and AdenoE1-LMP plus MVA-IiE1 heterologous prime-boost vaccine formulations on $\mathrm{CD}^{+}$and $\mathrm{CD} 8^{+} \mathrm{T}$ cell populations for protection from EL4-E1 challenge, either $\mathrm{CD}^{+}$or $\mathrm{CD}^{+} \mathrm{T}$ cells were depleted with Abs on 3 consecutive days before the prime and the boost. Two weeks after the boost, $2 \times 10^{5}$ EL4-E1 cells were injected s.c. and measured every second day by caliper (Figure 5A). We confirmed complete $\mathrm{T}$ cell depletion in blood on the day of the prime and the boost and also observed a substantial decrease in the respective $\mathrm{T}$ cell populations of splenic cells, even around 45 days after the last depleting Ab injection (Supplemental Figure 3C). Notably, the $\mathrm{T}$ cell-depleted mice in the PBS treatment group did not show a significant difference of EL4-E1 tumor growth kinetics compared with the nondepleted animals (Figure 5B). When we compared the survival of depleted versus nondepleted mice, we noted a trend toward early dropouts in the tumor cell-challenged and $\mathrm{CD}^{+}$or $\mathrm{CD}^{+} \mathrm{T}$ cell-depleted mice without vaccination (Figure $5 \mathrm{C})$. Following $\mathrm{CD} 4^{+} \mathrm{T}$ cell depletion, both heterologous vaccines lost the ability to control EL4-E1 tumor growth. Loss of tumor growth control, to a lesser extent, was also observed in the CD8 ${ }^{+}$ $\mathrm{T}$ cell-depleted vaccinated animals. Moreover, the survival of $\mathrm{T}$ cell-depleted vaccinated mice was drastically diminished in comparison with the survival of nondepleted vaccinated mice. Even though vaccinated and $\mathrm{CD} 8^{+} \mathrm{T}$ cell-depleted mice still maintained some immune control of tumor growth (Figure 5B), their survival was significantly reduced (Figure 5C), possibly due to immunopathology of more strongly stimulated EBNA1-specific CD4 ${ }^{+} \mathrm{T}$ cells. In order to assess whether $\mathrm{CD}^{+} \mathrm{T}$ cell help is required for comprehensive $\mathrm{CD}^{+} \mathrm{T}$ cell priming and maintenance, splenic cells were restimulated with the EBNA1 peptide library at sacrifice, and $\mathrm{CD}^{+} \mathrm{T}$ cell responses were measured by ICS for IFN- $\gamma$ secretion (Figure 5D). We observed a diminished EBNA1-specific CD8 ${ }^{+} \mathrm{T}$ cell response after $\mathrm{CD} 4^{+} \mathrm{T}$ cell depletion in mice vaccinated with $\alpha$ DEC-E1 plus Adeno-E1-LMP in comparison with the mice in the nondepleted group. This trend was also visible, but not significant, in mice in the Adeno-E1-LMP plus MVA-IiE1 vaccination group. In addition, we found abrogated $\alpha$ EBNA1 Ab titer in the heterologously vaccinated mice after $\mathrm{CD} 4^{+} \mathrm{T}$ cell depletion (Supplemental Figure 3E). To understand the importance of peripheral $\mathrm{T}$ cell immunity for EL4-E1 metastasis, LNs were taken and analyzed for EBNA1 DNA content by quantitative PCR (qPCR) normalized to ubiquitin $\mathrm{C}(U B C)$. In general, all tested vaccinations reduced the number of mice with LN metastasis. However, this control was strongly decreased upon $\mathrm{CD} 8^{+} \mathrm{T}$ cell depletion in mice vaccinated with $\alpha$ DEC-E1 plus Adeno-E1-LMP, whereas depletion of both T cell subsets in the Adeno-E1-LMP plus MVA-IiE1 group led to a higher percentage of mice with LN metastasis (Figure 5E). Altogether, this suggests that after heterologous prime-boost vaccination, the main EL4-E1 tumor site is controlled primarily by $\mathrm{CD}^{+}$ $\mathrm{T}$ cell-dependent processes, whereas control over the spreading of tumor cells toward other organs mainly relies on peripheral EBNA1-specific CD8 ${ }^{+} \mathrm{T}$ cell immunity. We also analyzed the amount of EBNA1 DNA in the isolated tumors after the depletion experiments and found that relapsing tumors after successful treatment with heterologous prime-boost vaccinations such as aDEC-E1 plus Adeno-E1-LMP and Adeno-E1-LMP plus MVA-IiE1 lost the EBNA1 DNA almost completely (Supplemental Figure 3F). This might have occurred as a result of the strategy of generating EL4-E1 by sorting for $\mathrm{EBNA1}^{+} \mathrm{GFP}^{+}$cells after lentiviral transduction, which yielded purities of only approximately $98 \%$. The negative selection pressure on EBNA1 ${ }^{+}$EL 4 cells might be very high during the vaccinations, with the result that the remaining $2 \%$ EBNA1 ${ }^{-}$EL4 cells survive and relapse. These studies demonstrate a dependence on both $\mathrm{CD}^{+}$and $\mathrm{CD}^{+} \mathrm{T}$ cells for protection from EL4-E1 tumor challenge after heterologous vaccination.

Protection from EBNA1-induced B cell lymphoma challenge by heterologous vaccination. To test the most promising heterologous prime-boost vaccinations against a tumor model that more closely resembles human EBV-associated malignancies, especially c-Myc-driven Burkitt's lymphoma, we used EBNA1-induced B lymphoma cells on a C57BL/6 background (36). These EBNA1 ${ }^{+}$ 
A

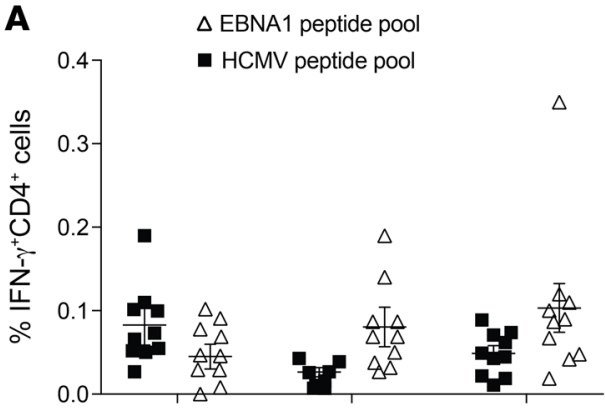

B

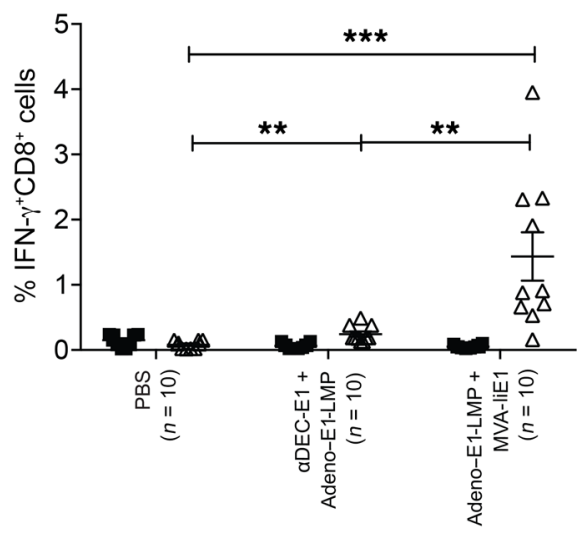

C

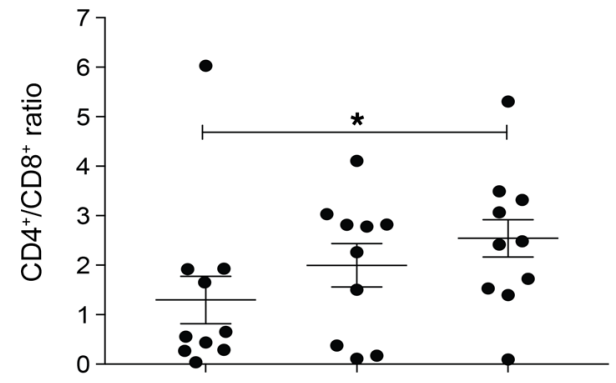

D $\square$ Treatment + tumor $(n=10)$
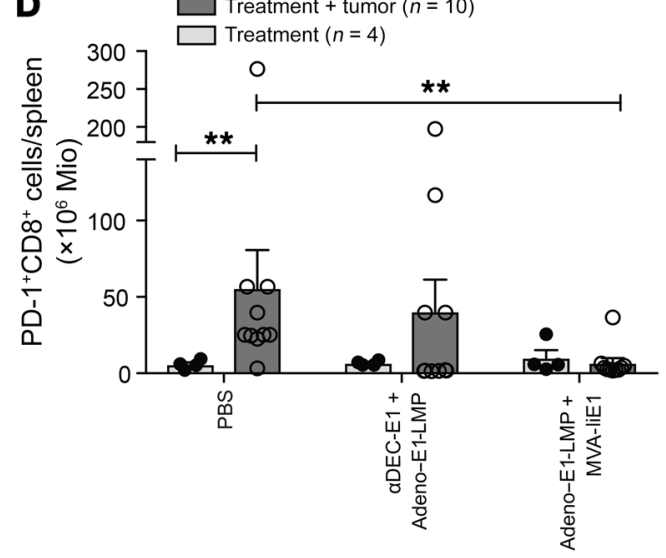

$\mathbf{E}$
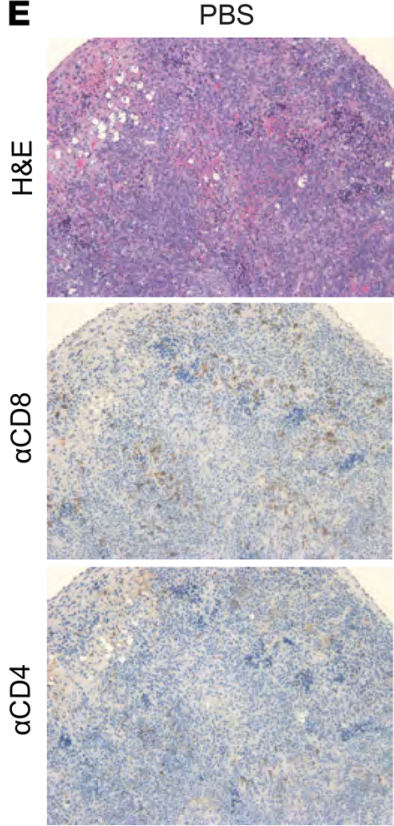

aDEC-E1 + Adeno-E1-LMP
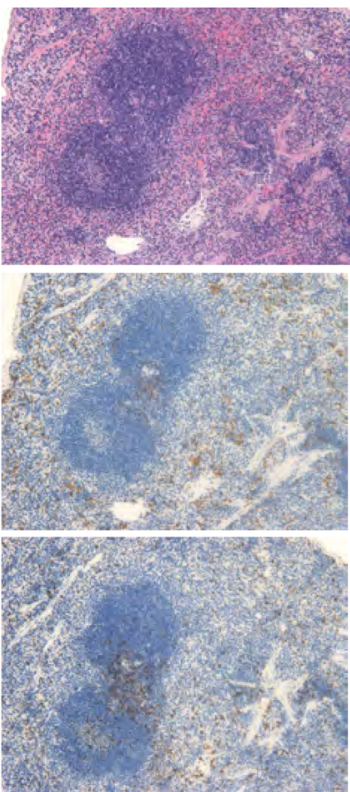

Adeno-E1-LMP + MVA-liE1
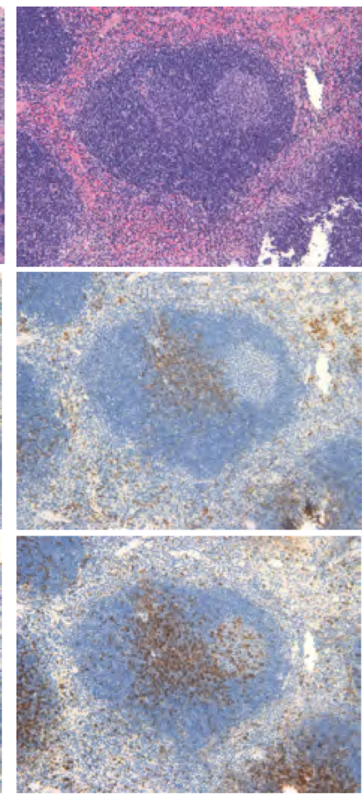

PBS, no tumor

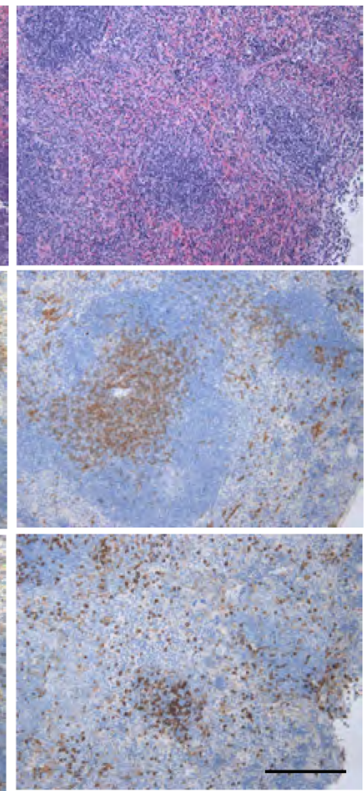

Figure 7. Characteristics of T cell responses toward EBNA1-induced B cell lymphomas with and without protective vaccination. huDEC205-Tg mice were immunized with different combinations of vaccines for the prime and the boost, scheduled 10 days apart. Mice in the preventive group were challenged i.v. with $3 \times 10^{6}$ to $5 \times 10^{6} \mathrm{BL}$-E1 tumor cells 14 days after the boost. (A) At sacrifice, bulk splenocytes were harvested and stimulated with $1 \mu \mathrm{g} / \mathrm{ml}$ EBNA1 or control HCMV pp65 peptide pools. IFN- $\gamma$ production was monitored by ICS in CD4+ gated cells. Data are shown as the mean \pm SEM from 2 independent experiments with at least 5 mice per group. Statistical analyses was done using a 2-tailed Mann-Whitney $U$ test. (B) After splenocyte stimulation, IFN- $\gamma$ production was monitored by ICS in $\mathrm{CD} 8{ }^{+}$gated cells. Data are shown as the mean $\pm \mathrm{SEM}$ from 2 independent experiments with at least 5 mice per group. ${ }^{* *} P<0.01$ and ${ }^{* * *} P<0.001$; Kruskal-Wallis with Dunn's multiple comparisons test (C) The $\mathrm{CD} 4^{+} / \mathrm{CD} 8^{+} \mathrm{T}$ cell ratio was calculated using the percentages of each subset in the spleen. ${ }^{*} P<0.05 ; 1$-way ANOVA with Tukey's multiple comparisons test. (D) At sacrifice, bulk splenocytes were harvested and stained for PD- 1 on CD8 $8^{+}$gated cells. Total PD-1+CD8 ${ }^{+}$cell amounts per spleen were calculated using the total splenocytes count. Data are shown as the mean \pm SEM from 2 independent experiments with at least 5 mice per group. Mice with PBS treatment or vaccination and tumor injection were compared with mice that were only treated with PBS or vaccinated. ${ }^{* *} P<0.01 ;$ Kruskal-Wallis with Dunn's multiple comparisons test. (E) Splenic tissue was fixed in PFA and embedded in paraffin and then stained with H\&E, $\alpha \mathrm{CD} 8$, and $\alpha \mathrm{CD} 4$. One representative image from each group is shown, along with an image of splenic cells from a PBS-treated mouse without tumor challenge. Scale bar: $20 \mu \mathrm{m}$. 
B lymphoma cells (BL-E1) occur spontaneously in the LNs and spleens of E $\mu$ EBNA1-Tg mice (37) and show relatively low EBNA1 expression, which can be visualized by Western blotting but not IHC (Supplemental Figure 4A). BL-E1 tumor cells overexpress the c-Myc proto-oncogene, as do Burkitt's lymphomas. Tumorigenesis was found to be unequivocally linked to EBNA1 expression and dependent not only on c-Myc but on Mdm2 deregulation as well (36). To evaluate the protective value of the vaccinations against these EBNA1-induced B cell lymphomas, $3 \times 10^{6}$ to $5 \times 10^{6} \mathrm{CD}^{+} 9^{+}$ $\mathrm{B}$ cells isolated from the spleens of tumor-bearing E $\mu$ EBNA1-Tg mice were injected i.v. 14 days after the boost. The mice were euthanized at the latest 45 days after tumor cell injection or when they showed signs of sickness such as weight loss or reduced activity (Figure 6A). At sacrifice, DNA from the spleen, blood, LNs, and liver was isolated and analyzed for EBNA1 DNA levels (Figure 6B). Following Adeno-E1-LMP plus MVA-IiE1 vaccination, we found that the amount of EBNA1 DNA was lower in all analyzed organs when compared with levels in PBS-treated mice, whereas in the $\alpha$ DEC-E1 plus Adeno-E1-LMP group, the levels were similar to those in the PBS-treated control group. We evaluated the total tumor burden in each mouse using the detection limit of EBNA1 DNA qPCR (Figure 6C). After Adeno-E1-LMP plus MVA-IiE1 vaccination, over half of the mice remained tumor free in all of the organs examined, whereas only $35 \%$ of the mice vaccinated with $\alpha \mathrm{DEC}-\mathrm{E} 1$ plus Adeno-E1-LMP were tumor free. Forty-five percent of the PBS-treated mice had BL-E1 metastasis in 3 or more of the analyzed organs, whereas none of the mice treated with AdenoE1-LMP plus MVA-IiE1 had metastasis in more than 2 organs. The phenotype of EBNA1-induced B cell lymphomas was studied previously (36). In our study, we confirmed that expression of XIAP and proliferating cell nuclear antigen (PCNA), a proliferation marker like Ki67, by IHC, and expression of EBNA1, by Western blotting, were strongly correlated with tumor pathology in the mice (Figure 6D and Supplemental Figure 4A). In vaccinated mice, only CD19hi-expressing cells with a more typical lymphocyte morphology could be found, which indicates that these cells are classical B cells. Following EBNA1 ${ }^{+}$tumor cell injection, CD19 dim_ expressing cells in PBS-treated mice accumulated with PCNA and XIAP expression compared with mice treated with $\alpha$ DEC-E1 plus Adeno-E1-LMP or Adeno-E1-LMP plus MVA-IiE1. The expression of XIAP was not unexpected, since it was shown that EBNA1 tumorigenesis is dependent on $\mathrm{Mdm} 2$ signaling, which promotes XIAP translation (36). Taken together, we conclude that $\alpha \mathrm{DEC}-\mathrm{E} 1$ plus Adeno-E1-LMP vaccination seems to lower the tumor burden upon BL-E1 injection, whereas Adeno-E1-LMP plus MVA-IiE1 vaccination leads to a more effective reduction in tumor load, as indicated by the EBNA1 DNA load, spleen histology, and EBNA1specific Western blot analysis.

Characteristics of $T$ cell responses to EBNA1-induced B cell lymphomas with and without protective vaccination. In order to investigate the different mechanisms of the vaccination strategies used to restrict EBNA1-induced B cell lymphomas, we further assessed T cell populations by FACS and histological analyses. At sacrifice, splenic cells were restimulated with EBNA1 and HCMV control peptide libraries, and $\mathrm{CD}^{+}$and $\mathrm{CD} 8^{+} \mathrm{T}$ cell responses were measured by IFN- $\gamma$ secretion with ICS. We observed no difference in the percentage of IFN- $\gamma$-secreting $\mathrm{CD}^{+} \mathrm{T}$ cells in vaccinated mice compared with the percentage in PBS-treated mice (Figure 7A). However, the percentage of EBNA1-specific IFN- $\gamma$-secreting $\mathrm{CD}^{+} \mathrm{T}$ cells was significantly enhanced after Adeno-E1-LMP plus MVA-IiE1 vaccination (Figure 7B). Curiously, $\alpha$ DEC-E1 plus Adeno-E1-LMP vaccination led to only a modest increase in EBNA1-specific CD8 ${ }^{+} \mathrm{T}$ cells. In recent studies, it was shown that mice with primary BL-E1 tumors had an imbalanced $\mathrm{CD} 4^{+} / \mathrm{CD}^{+}$ $\mathrm{T}$ cell ratio in the spleen, which was lower compared with mice without tumors (36). We observed a slight decrease of both CD4 ${ }^{+}$ and $\mathrm{CD} 8^{+} \mathrm{T}$ cells in tumor-bearing spleens compared with spleens in tumor-free mice, and this was most visible in the PBS-treated mice (Supplemental Figure 4B). The different levels of $\mathrm{CD}^{+}$and $\mathrm{CD}^{+} \mathrm{T}$ cells in the $\mathrm{T}$ cell compartments were also observed in the ratio of $\mathrm{CD}^{+}$to $\mathrm{CD} 8^{+} \mathrm{T}$ cells, which was significantly decreased in the PBS-treated group (Figure $7 \mathrm{C}$ ). The inability of $\mathrm{CD}^{+} \mathrm{T}$ cells to respond to EBNA1 antigen restimulation in the group of mice vaccinated with $\alpha$ DEC-E1 plus Adeno-E1-LMP raised the question of whether those $\mathrm{T}$ cells showed upregulation of programmed cell death protein 1 (PD-1), which is known to play a role in attenuating tumor immunity in many different types of cancers. Indeed, PD-1 levels were substantially increased on splenic CD8 ${ }^{+} \mathrm{T}$ cells from PBS-treated mice that underwent tumor cell injection compared with levels in healthy mice (Figure 7D). PD-1 expression on CD8 ${ }^{+}$ $\mathrm{T}$ cells after $\alpha \mathrm{DEC}-\mathrm{E} 1$ plus Adeno-E1-LMP vaccination reached an intermediate level, which was significantly lower than that in the tumor-bearing PBS-treated animals. Animals vaccinated with Adeno-E1-LMP plus MVA-IiE1 had very low PD-1 expression levels in their splenic CD8 ${ }^{+} \mathrm{T}$ cell compartment, independent of tumor cell injection. In order to examine the distribution of $\mathrm{T}$ cells in the affected organs, splenic sections were stained with $\mathrm{H} \& \mathrm{E}$ and $\alpha \mathrm{CD} 4$ and $\alpha \mathrm{CD} 8$ Abs (Figure $7 \mathrm{E}$ ). Although most mice that were both $\mathrm{PBS}$ treated and tumor challenged showed disruption of the white pulp and T cell zones, $\alpha$ DEC-E1 plus Adeno-E1-LMP vaccination could attenuate this phenotype, leading to small $\mathrm{T}$ cell zones and differentiation of white and red pulp in some areas. In contrast, most mice in the Adeno-E1-LMP plus MVA-IiE1 vaccination group had spleens with a healthy phenotype, sharp separation of red and white pulp, and large $\mathrm{T}$ cell zones, all of which were observed in PBS-treated mice without tumor challenge. Liver sections from PBS-treated mice confirmed these alterations after BL-E1 tumor establishment, which led to high lymphocyte infiltration and structural damage in the livers of tumor-bearing mice (Supplemental Figure $4 \mathrm{C}$ ). Whereas $\alpha$ DEC-E1 plus AdenoE1-LMP seemed to have a similarly strong effect on EBNA1 ${ }^{+} \mathrm{T}$ cell lymphomas, these findings suggest that Adeno-E1-LMP plus MVA-IiE1 vaccination might be much more suitable for preventing EBV-associated B cell malignancies.

\section{Discussion}

Our study identifies heterologous prime-boost regimens of preferentially $\mathrm{CD}^{+}$and $\mathrm{CD} 8^{+} \mathrm{T}$ cell-priming vaccine formulations as superior immunization strategies to expand EBNA1-specific $\mathrm{CD}^{+}$and $\mathrm{CD}^{+} \mathrm{T}$ cell responses, with EBNA1-expressing adenovirus boosted by MVA vaccination being the most promising approach. These provide protection against EBV antigen-expressing $\mathrm{T}$ and $\mathrm{B}$ cell lymphomas, the latter of which spontaneously originated from Tg EBNA1 expression in murine 
B cells and share some similarities with EBV-associated B cell lymphomas in humans, primarily latency I Burkitt's lymphomas $(36,37)$. Therefore, EBNA1-based heterologous prime-boost vaccinations should be further developed as therapeutic strategies against EBV-associated malignancies.

In contrast, homologous vaccinations with EBNA1-encoding recombinant viral vectors have already been attempted in patients with nasopharyngeal carcinoma $(38,39)$. In these studies, a recombinant MVA vector was used that encodes both EBNA1 and LMP2 and is capable of expanding specific CD4 $4^{+}$and CD8 ${ }^{+}$ $\mathrm{T}$ cells in response to these 2 viral antigens in vitro (25). Intradermal injection of this vaccine candidate increased EBNA1- and/or LMP2-specific T cell responses in 15 of 18 treated Chinese and in 8 of 14 British patients with nasopharyngeal carcinoma $(38,39)$. Furthermore, this vaccination increased the proportion of these 2 viral antigen-specific T cells, which produced TNF- $\alpha$, IFN- $\gamma$, and/ or IL-2, suggesting the functional superiority of these T cells (39). In parallel, in another study, a recombinant adenoviral vector encoding EBNA1 and HLA-A2-restricted polyepitopes of LMP1 and LMP2 was explored (40). In vitro stimulation with this vaccine formulation reversed the functional impairment of EBV-specific $\mathrm{CD}^{+} \mathrm{T}$ cells from patients with Hodgkin's lymphoma (26). Moreover, in vitro expansion of EBNA1- and LMP-specific T cells in vitro and adoptive transfer into patients with nasopharyngeal carcinoma after primary tumor resection more than doubled the patients' median overall survival (41). As in our mouse model, a balanced expansion of EBV-specific $\mathrm{CD}^{+}$and $\mathrm{CD}^{+} \mathrm{T}$ cell responses was suggested to be important for these clinical effects. Previous studies in patients with nasopharyngeal carcinoma showed that only transiently expanded $\mathrm{CD} 8^{+} \mathrm{T}$ cells with LMP2 peptide-loaded or LMP1- as well as LMP2-encoding adenovirus-infected DCs led to partial clinical responses in only 2 of 16 and 1 of 12 of the patients (42-44). We explored new vaccination strategies, and our findings suggest that improved $\mathrm{CD}^{+}$and $\mathrm{CD}^{+} \mathrm{T}$ cell-mediated EBV immune control might be achieved by heterologous prime-boost vaccinations with EBNA1 as the protective EBV antigen.

Heterologous prime-boost vaccination strategies combine different antigen delivery systems to improve immune responses. Our in vitro studies compared targeting of 8 different surface receptors using EBNA1-specific $\mathrm{CD}^{+}$and $\mathrm{CD} 8^{+} \mathrm{T}$ cell clones as the readout for the efficiency of antigen presentation in PBMCs. DEC205 remains one of the most efficient targeted receptors for stimulating $\mathrm{T}$ cell responses (Figure 1). This is consistent with other studies using different methods to assess the level of antigen presentation (45). However, vaccination by antigen targeting to the DC receptor DEC205 elicits, with the exception of hen egg-derived model antigens, mostly $\mathrm{CD} 4^{+} \mathrm{T}$ cell responses in vivo $(21,22,46-54)$. This $\mathrm{CD} 4^{+} \mathrm{T}$ cell bias also led to only modest efficacy after DEC205-targeted NY-ESO1 vaccination, with tumor regression seen in only 2 of 45 patients (55). These CD $4^{+}$ $\mathrm{T}$ cell responses could, however, be complemented with $\mathrm{CD}^{+} \mathrm{T}$ cell responses by a heterologous poxvirus-based vaccination for HIV gag p24 in nonhuman primates (56). Expanding on this previous study further, we compared the boosting of 3 different viral vectors after priming with DEC205 and showed that priming with DEC205 targeting and boosting with either adenoviral or lentiviral vector vaccines, but not boosting with MVA, resulted in a robust antigen-specific $\mathrm{CD}^{+} \mathrm{T}$ cell response (Figure 2 and Figure 3). Moreover, the improved $\mathrm{CD} 4^{+}$and $\mathrm{CD} 8^{+} \mathrm{T}$ cell responses following heterologous prime-boost vaccination with $\alpha \mathrm{DEC}-\mathrm{E} 1$ and Adeno-E1-LMP were translated into protection against EBV antigen-expressing lymphoma challenge.

In parallel to this development of heterologous prime-boost vaccinations with DC-targeted antigens, heterologous primeboost vaccinations with different viral vectors were developed. Originally designed to confer both $\mathrm{CD} 8^{+} \mathrm{T}$ cell-mediated immune control of the liver stage and $\mathrm{CD} 4^{+} \mathrm{T}$ cell-orchestrated immune suppression of the blood stage of malaria infection (57), heterologous adeno- and poxvirus vaccination was used in clinical trials for malaria, Ebola, and influenza virus antigens $(28,58-$ 60). $\mathrm{CD}^{+} \mathrm{T}$ cell-dependent Ab production was mainly observed after poxvirus vaccination, whereas adenovirus-derived antigen expression allowed for $\mathrm{CD} 8^{+} \mathrm{T}$ cell priming. This optimized $\mathrm{CD}^{+}$and $\mathrm{CD}^{+} \mathrm{T}$ cell vaccination regime reduced malaria infection by two-thirds in African adults (28) and established protection against EBV antigen-expressing $\mathrm{T}$ and $\mathrm{B}$ cell lymphomas in our study. Interestingly, $\alpha$ DEC-E1 plus Adeno-E1-LMP had protective efficacy against $\mathrm{T}$ cell lymphoma that was equivalent to that of Adeno-E1 plus MVA-IiE1 vaccination (Figure 4). Also, protein vaccines have the advantage of being more readily manufactured, safe, and less expensive than viral vector vaccines. The 2 clinical settings in which such vaccination strategies could be tested include the treatment of EBV-seronegative adolescents with a $30 \%-50 \%$ risk for developing infectious mononucleosis upon primary EBV infection (61) and patients with EBV-associated lymphomas or carcinomas, the latter of which are the most frequent EBV-associated malignancies with currently limited therapeutic options (3). These vaccination strategies might be less useful in patients with EBV-associated lymphomas that emerge during immune suppression, as these lymphomas can be successfully targeted by adoptive EBV-specific T cell transfer (11). Thus, we plan to further develop heterologous $\alpha$ DEC-E1 plus Adeno-E1-LMP and heterologous Adeno-E1 plus MVA-IiE1 vaccination strategies in order to improve therapeutic vaccination for patients with EBV-associated tumors as well as prophylactic vaccination to prevent the symptomatic primary EBV infection that leads to infectious mononucleosis.

\section{Methods}

aDEC205-EBNA1 and other EBNA1-Ab fusion proteins. $\alpha$ DEC205-EBNA1 fusion Abs were produced by transient transfection (calcium chloride) of human HEK293T cells. The fusion Abs were tested for binding as described previously (21). All other EBNA1-Ab fusion proteins were designed and produced in collaboration with Miltenyi Biotec. Abs were produced in stably transfected, nonadherent HEK293T cell lines and were purified using Protein L columns (GE Healthcare) for a first purification and high-performance HisTrap Nickel-NTA columns (GE Healthcare) for a second purification step. Dialysis was performed overnight in 1 liter of $1 \times$ PBS using dialysis tubing from Spectrum Labs (3.5 kDa molecular weight cutoff [MWCO]; Thermo Fisher Scientific). Characterization was done by SDS-PAGE followed by Western blotting with rat $\alpha E B N A 1$ primary $\mathrm{Ab}$ (clone $1 \mathrm{H} 4$ ), which was provided by Friedrich Grässer (62). Binding assays were performed with increasing concentrations of competitive pure Abs of the same clonal specificity. 
For the $\mathrm{Ab}$ fusion proteins, the following clones srom Miltenyi Biotec were used: BDCA1 (AD5-8E7), BDCA3 (AD5-14H12), CD40 (HB14), CD11c (MJ4-27G12), CD206 (DCN228), CD207 (MB22-9F5), HLADR (AC122), and DEC205 (MG38.2).

Viral vectors. The Adeno-E1-LMP recombinant adenoviral vector used in this study carries an EBNA1-LMP-polyepitope insert, which is incorporated into the replication-deficient mammalian vector Ad5F35, as previously described (26). The MVA is an attenuated vaccinia virus that has been used for smallpox vaccination (63). The MVA vector pSC11 carried a fusion protein insert of the Gly/ Ala repeat-deleted EBNA1, either with Ii (MVA-IiE1) or without it (MVA-E1) (25). MVA-IiE1 and MVA-E1 viruses were produced as previously described (25). Additionally, we used a replication-impaired lentivirus carrying EBNA1 with and without the invariant chain (Ii) in a pHR-SIN-CSGWDNotI (pCSGW) backbone with an IRES-GFPtag (referred to herein as Lenti-E1 and Lenti-IiE1), together with the 2 helper plasmids pMDG and pCMVDR8.91 (p8.9) (64). The invariant chain functions as a guiding protein for the EBNA1 protein and targets EBNA1 for the endolysosomal pathway for degradation. This facilitates the processing of EBNA1 and subsequent presentation on MHC class II. To produce Lenti-E1 and Lenti-IiE1, 107 HEK293T cells were transfected with the $20 \mu \mathrm{g}$ of the plasmid of interest and the 2 lentiviral packaging plasmids (10 $\mu \mathrm{g}$ pMDG and $20 \mu \mathrm{g}$ p8.9) using transient transfection with calcium chloride. Approximately 30 to 32 hours after medium exchange, the virus was harvested. The viral supernatant was collected, centrifuged, filtered, and purified using the Vivapure LentiSELECT 40 kit (Sartorius) according to the manufacturer's protocol. The purified virus was eluted into cold PBS, aliquoted, and stored at $-80^{\circ} \mathrm{C}$. Lenti-E1 and Lenti-IiE1 were titrated on HEK293T cells and incubated for 2 days. The amount of infected cells was quantified by GFP expression using a FACSCanto II system (BD Biosciences). The concentration of transfection units per milliliter $(\mathrm{TU} / \mathrm{ml})$ was calculated using the following formula: (\% infected cells $\times$ cells used in the titration $/ 100 \times 1000 \mu \mathrm{l} / \mu \mathrm{l}$ virus added to the well $)=\mathrm{TU} / \mathrm{ml}$.

Tumor models. EL4 cells were provided by Melanie Greter (University of Zurich). These EL4 cells were infected by Lenti-E1 GFP, single-cell sorted with a FACSAria III 5L at the University of Zurich Cytometry Core Facility, and reached a purity of $98 \% \mathrm{GFP}^{+}$cells. EBNA1 $^{+}$B lymphoma cells were harvested as previously described $(36,37)$. Both cell lines were analyzed by Western blotting for EBNA1 expression using rat $\alpha \mathrm{EBNA} 1$ primary $\mathrm{Ab}$ (clone $1 \mathrm{H} 4$, provided by Friedrich Grässer [ref. 62]; diluted 1:50 in PBS).

huDEC205-Tg mice. huDEC205-Tg C57BL/6 mice were a gift from Cheolho Cheong (IRCM, Montreal, Canada) and were bred at 8 to 12 weeks of age at the local animal facility of the University of Zurich. Maintenance of the huDEC205 transgene was controlled by PCR for each mouse using the forward primer $5^{\prime}$-TGGAAGAGACATGGAGAAACCT-3' and reverse primer 5'-TCTCAGGCCAGTCCAGAAGTA-3'.

$T$ cell assays. PBMCs were obtained from whole blood of donors after red blood cell removal by density gradient centrifugation using Ficoll-Paque (GE Healthcare) following the manufacturer's instructions. PBMCs were either incubated for 4 hours with $1 \mu \mathrm{g} / \mathrm{ml}$ EBNA1 fusion Abs or DMSO control or were infected with viral vectors at a MOI of 10 for 24, 48, 72, and 96 hours. As a positive control, PBMCs were incubated with $5 \mu \mathrm{M}$ cognate peptide for 1 hour. PBMCs were washed extensively with PBS and T cell assays were performed in duplicates by coculturing autologous EBNA1-loaded PBMCs $(5 \times$ $10^{4} /$ well) overnight with $\mathrm{T}$ cell clones $\left(5 \times 10^{3} /\right.$ well $)$ in 96 -well V-bottomed plates. IFN- $\gamma$ released into the supernatant was measured by IFN- $\gamma$ ELISA (Mabtech).

$\alpha E B N A 1 \operatorname{IgG}$ ELISA. The $\alpha$ EBNA1 IgG titer was acquired from serum samples at the point of sacrifice or from plasma acquired during bleeding procedures using the EBNA IgG ELISA kit (Bio-Rad) with goat $\alpha$-mouse-HRP conjugate diluted 1:2000 in PBS. The OD was measured at $450 \mathrm{~nm}$ by the TECAN Infinite M200 Pro microplate reader.

EBNA1 copy quantification by $q P C R$. DNA from single-cell suspensions of cells from blood, spleen, liver, and LNs was isolated using a DNA isolation kit (QIAGEN). qPCR was performed using $25 \mathrm{ng}$ of each sample in triplicates with the TaqMan Universal PCR kit from Applied Biosystems. The probe 5'-/56-FAM/AGGAACTGC/ZEN/CCTTGCTATTCCACA/3IBkFQ/-3', primer 5'-GGAGACGACTCAATGGTGTAAG-3' and 5'-GGTGTGTTCGTATATGGAGGTAG-3' from Integrated DNA Technologies was used for EBNA1 qPCR. EBNA1 abundance was normalized to the $U B C$ housekeeping gene with probe 5' -/56-FAM/CGAGCCCAG/ZEN/TGACACCATTGAGAA/3IBkFQ/-3', primer 5'-CCTCCTTGTCCTGGATCTTTG-3', and 5'-AGGTGGGATGCAGATCTTTG-3'.

Histology. Tissue was fixed using $4 \%$ formalin and then embedded in paraffin. Histological stainings were performed by Sophistolab. For IHC, 3- $\mu$ m sections were processed on a Leica BOND-MAX or Bond-III automated IHC system. Stainings were performed with monoclonal rat $\alpha$-mouse CD19 (clone 60MP31, Invitrogen, Thermo Fisher Scientific), rat $\alpha$-mouse CD4 (clone 4SM94, eBioscience), rat $\alpha$-mouse CD8 (clone 4SM15, eBioscience), rat $\alpha$ FoxP3 (clone EP340, Clinisciences), and $\alpha$ PCNA (clone PC10, BioLegend). EBNA1-specific IHC was performed with the $1 \mathrm{H} 4 \mathrm{Ab}$ as previously described (64).

In vivo immunization. Mice were injected i.p. with $5 \mu \mathrm{g} \alpha$-mouse DEC205 fused with EBNA1 mAb with $50 \mu \mathrm{g}$ poly(I:C)-LMW (InvivoGen) as an adjuvant (21) or i.v. with viral vectors at different infectious units. The adenoviral vector was administered at $10^{9} \mathrm{PFU} /$ mouse (40), whereas all other viral vectors were injected at $1.5 \times 10^{7} \mathrm{TU} /$ mouse. The immunization was boosted 10-14 days later with the same dose of $\alpha$ DEC205-E1/poly(I:C) or a viral vector. One week after the boost, the mice were sacrificed, and bulk splenocytes were isolated for analysis.

In vivo T cell depletion. T cell depletion was done by injections of 200 $\mu \mathrm{g} \alpha \mathrm{CD} 4 \mathrm{mAb}$ (GK1.5) or $\alpha \mathrm{CD} 8 \mathrm{mAb}$ (2.43) (commercially available from Bio X Cell) on 3 consecutive days before the prime and the boost.

Statistics. Statistical analysis was performed using 1-way ANOVA with Bonferroni's pre-test, 2-way ANOVA with Tukey's multiple comparisons test, Kruskal-Wallis with Dunn's post test, Mantel-Cox, or 2-tailed Mann-Whitney $U$ test. $P$ values below 0.05 were considered statistically significant.

Study approval. All animal protocols were approved by the cantonal veterinary office of the canton of Zurich, Switzerland (protocols 209/2014 and 159/2017). All studies involving human samples were reviewed and approved by the cantonal ethics committee of Zurich, Switzerland (protocol KEK-StV-Nr.19/08).

\section{Author contributions}

JR, CSL, and CM conceived and designed the experiments. JR, CC, and CE performed the experiments. JR, CSL, and CC analyzed the data. RK, GST, JBW, AD, TH, and JD contributed reagents and materials. JR, CSL, and CM wrote the manuscript. 


\section{Acknowledgments}

This study was supported by Cancer Research Switzerland (KFS4091-02-2017); the clinical research priority programs KFSP ${ }^{\mathrm{MS}}$ and KFSPHHLD of the University of Zurich; the Vontobel Foundation; the Baugarten Foundation, the Sobek Foundation; the Swiss Vaccine Research Institute; the Swiss MS Society; the Swiss National Science Foundation (310030_162560 and CRSII3_160708); and Worldwide Cancer Research (AICR 11-0516 and WCR 14-1033).
Address correspondence to: Carol Leung, Ludwig Institute for Cancer Research, University of Oxford, Nuffield Department of Medicine, Old Road Campus Research Building, Roosevelt Drive, Oxford OX3 7DQ, United Kingdom. Phone: 44.1865.617507; Email: carol.leung@ludwig.ox.ac.uk. Or to: Christian Münz, Institute of Experimental Immunology, University of Zurich, Winterthurerstrasse 190, 8057 Zürich, Switzerland. Phone: 41.44.635.3716; Email: christian.muenz@uzh.ch.
1. Young LS, Yap LF, Murray PG. Epstein-Barr virus: more than 50 years old and still providing surprises. Nat Rev Cancer. 2016;16(12):789-802.

2. Cesarman E. Gammaherpesviruses and lymphoproliferative disorders. Annu Rev Pathol. 2014;9:349-372.

3. Cohen JI, Fauci AS, Varmus H, Nabel GJ. EpsteinBarr virus: an important vaccine target for cancer prevention. Sci Transl Med. 2011;3(107):107fs7.

4. Babcock GJ, Hochberg D, Thorley-Lawson AD. The expression pattern of Epstein-Barr virus latent genes in vivo is dependent upon the differentiation stage of the infected B cell. Immunity. 2000;13(4):497-506.

5. Babcock GJ, Decker LL, Volk M, Thorley-Lawson DA. EBV persistence in memory B cells in vivo. Immunity. 1998;9(3):395-404.

6. Tangye SG, Palendira U, Edwards ES. Human immunity against EBV-lessons from the clinic. J Exp Med. 2017;214(2):269-283.

7. Totonchy J, Cesarman E. Does persistent HIV replication explain continued lymphoma incidence in the era of effective antiretroviral therapy? Curr Opin Virol. 2016;20:71-77.

8. Cohen JI. Primary immunodeficiencies associated with EBV disease. Curr Top Microbiol Immunol. 2015;390(Pt 1):241-265.

9. Taylor GS, Long HM, Brooks JM, Rickinson AB, Hislop AD. The immunology of Epstein-Barr virus-induced disease. Annu Rev Immunol. 2015;33:787-821.

10. Münz C. Epstein-Barr Virus-Specific Immune Control by Innate Lymphocytes. Front Immunol. 2017;8:1658.

11. McLaughlin LP, Gottschalk S, Rooney CM, Bollard CM. EBV-Directed T Cell Therapeutics for EBV-Associated Lymphomas. Methods Mol Biol. 2017;1532:255-265.

12. Icheva $V$, et al. Adoptive transfer of epstein-barr virus (EBV) nuclear antigen 1-specific t cells as treatment for EBV reactivation and lymphoproliferative disorders after allogeneic stem-cell transplantation. J Clin Oncol. 2013;31(1):39-48.

13. Münz C, et al. Human CD4(+) T lymphocytes consistently respond to the latent EpsteinBarr virus nuclear antigen EBNA1. J Exp Med. 2000;191(10):1649-1660.

14. Long HM, et al. CD4 ${ }^{+} \mathrm{T}$-cell responses to EpsteinBarr virus (EBV) latent-cycle antigens and the recognition of EBV-transformed lymphoblastoid cell lines. J Virol. 2005;79(8):4896-4907.

15. Paludan C, et al. Epstein-Barr nuclear antigen 1-specific CD4(+) Th1 cells kill Burkitt's lymphoma cells. J Immunol. 2002;169(3):1593-1603.

16. Nikiforow S, Bottomly K, Miller G, Münz C. Cytolytic CD4(+)-T-cell clones reactive to EBNA1 inhibit Epstein-Barr virus-induced B-cell prolif- eration. J Virol. 2003;77(22):12088-12104.

17. Blake $\mathrm{N}$, et al. Human $\mathrm{CD}^{+} \mathrm{T}$ cell responses to EBV EBNA1: HLA class I presentation of the (Gly-Ala)-containing protein requires exogenous processing. Immunity. 1997;7(6):791-802.

18. Paludan C, et al. Endogenous MHC class II processing of a viral nuclear antigen after autophagy. Science. 2005;307(5709):593-596.

19. Leung CS, Haigh TA, Mackay LK, Rickinson AB, Taylor GS. Nuclear location of an endogenously expressed antigen, EBNA1, restricts access to macroautophagy and the range of CD4 epitope display. Proc Natl Acad Sci US A. 2010;107(5):2165-2170.

20. Kutok JL, Wang F. Spectrum of Epstein-Barr virus-associated diseases. Annu Rev Pathol. 2006;1:375-404.

21. Gurer C, et al. Targeting the nuclear antigen 1 of Epstein-Barr virus to the human endocytic receptor DEC-205 stimulates protective T-cell responses. Blood. 2008;112(4):1231-1239.

22. Meixlsperger S, et al. CD $141^{+}$dendritic cells produce prominent amounts of IFN- $\alpha$ after dsRNA recognition and can be targeted via DEC-205 in humanized mice. Blood. 2013;121(25):5034-5044

23. Leung CS, et al. Robust T-cell stimulation by Epstein-Barr virus-transformed B cells after antigen targeting to DEC-205. Blood. 2013;121(9):1584-1594.

24. Ngu LN, et al. In vivo targeting of protein antigens to dendritic cells using anti-DEC-205 single chain antibody improves HIV Gag specific $\mathrm{CD}^{+}{ }^{+} \mathrm{T}$ cell responses protecting from airway challenge with recombinant vaccinia-gag virus [published online ahead of print (March 13, 2017)]. Immun Inflamm Dis. https://doi.org/10.1002/iid3.151.

25. Taylor GS, et al. Dual stimulation of Epstein-Barr Virus (EBV)-specific CD4+- and CD8 ${ }^{+}$-T-cell responses by a chimeric antigen construct: potential therapeutic vaccine for EBV-positive nasopharyngeal carcinoma. J Virol. 2004;78(2):768-778.

26. Smith C, et al. Functional reversion of antigen-specific $\mathrm{CD}^{+} \mathrm{T}$ cells from patients with Hodgkin lymphoma following in vitro stimulation with recombinant polyepitope. JImmunol. 2006;177(7):4897-4906.

27. Milone MC, O'Doherty U. Clinical use of lentiviral vectors. Leukemia. 2018;32(7):1529-1541.

28. Ogwang C, et al. Prime-boost vaccination with chimpanzee adenovirus and modified vaccinia Ankara encoding TRAP provides partial protection against Plasmodium falciparum infection in Kenyan adults. Sci Transl Med. 2015;7(286):286re5

29. Manusama ER, Stavast J, Durante NM, Marquet RL, Eggermont AM. Isolated limb perfusion with
TNF alpha and melphalan in a rat osteosarcoma model: a new anti-tumour approach. Eur J Surg Oncol. 1996;22(2):152-157.

30. Wrangle JM, et al. IL-2 and Beyond in Cancer Immunotherapy. J Interferon Cytokine Res. 2018;38(2):45-68.

31. Bennett SR, Carbone FR, Karamalis F, Flavell RA, Miller JF, Heath WR. Help for cytotoxic-T-cell responses is mediated by CD 40 signalling. Nature. 1998;393(6684):478-480.

32. Ridge JP, Di Rosa F, Matzinger P. A conditioned dendritic cell can be a temporal bridge between a CD4 $4^{+}$T-helper and a T-killer cell. Nature. 1998;393(6684):474-478.

33. Schoenberger SP, Toes RE, van der Voort EI, Offringa R, Melief CJ. T-cell help for cytotoxic T lymphocytes is mediated by CD40-CD40L interactions. Nature. 1998;393(6684):480-483.

34. Fröhlich A, et al. IL-21R on T cells is critical for sustained functionality and control of chronic viral infection. Science. 2009;324(5934):1576-1580.

35. Williams MA, Tyznik AJ, Bevan MJ. Interleukin-2 signals during priming are required for secondary expansion of $\mathrm{CD}^{+}$memory T cells. Nature. 2006;441(7095):890-893.

36. AlQarni S, et al. Lymphomas driven by Epstein-Barr virus nuclear antigen-1 (EBNA1) are dependant upon Mdm2. Oncogene. 2018;37(29):3998-4012.

37. Wilson JB, Bell JL, Levine AJ. Expression of Epstein-Barr virus nuclear antigen-1 induces $\mathrm{B}$ cell neoplasia in transgenic mice. EMBO J. 1996;15(12):3117-3126.

38. Hui EP, et al. Phase I trial of recombinant modified vaccinia ankara encoding Epstein-Barr viral tumor antigens in nasopharyngeal carcinoma patients. Cancer Res. 2013;73(6):1676-1688.

39. Taylor GS, et al. A recombinant modified vaccinia ankara vaccine encoding Epstein-Barr Virus (EBV) target antigens: a phase I trial in UK patients with EBV-positive cancer. Clin Cancer Res. 2014;20(19):5009-5022.

40. Duraiswamy J, et al. Induction of therapeutic T-cell responses to subdominant tumor-associated viral oncogene after immunization with replication-incompetent polyepitope adenovirus vaccine. Cancer Res. 2004;64(4):1483-1489.

41. Smith C, et al. Effective treatment of metastatic forms of Epstein-Barr virus-associated nasopharyngeal carcinoma with a novel adenovirusbased adoptive immunotherapy. Cancer Res. 2012;72(5):1116-1125.

42. Lin CL, et al. Immunization with Epstein-Barr Virus (EBV) peptide-pulsed dendritic cells induces functional $\mathrm{CD} 8^{+} \mathrm{T}$-cell immunity and may lead to tumor regression in patients with EBV-positive nasopharyngeal carcinoma. Cancer Res. 2002;62(23):6952-6958. 
43. Li F, Song D, Lu Y, Zhu H, Chen Z, He X. Delayedtype hypersensitivity (DTH) immune response related with EBV-DNA in nasopharyngeal carcinoma treated with autologous dendritic cell vaccination after radiotherapy. J Immunother. 2013;36(3):208-214.

44. Chia WK, et al. A phase II study evaluating the safety and efficacy of an adenovirus- $\triangle$ LMP1-LMP2 transduced dendritic cell vaccine in patients with advanced metastatic nasopharyngeal carcinoma. Ann Oncol. 2012;23(4):997-1005.

45. Reuter A, et al. Criteria for dendritic cell receptor selection for efficient antibody-targeted vaccination. JImmunol. 2015;194(6):2696-2705.

46. Dudziak D, et al. Differential antigen processing by dendritic cell subsets in vivo. Science. 2007;315(5808):107-111.

47. Bonifaz LC, et al. In vivo targeting of antigens to maturing dendritic cells via the DEC-205 receptor improves $\mathrm{T}$ cell vaccination. J Exp Med. 2004;199(6):815-824.

48. Trumpfheller $\mathrm{C}$, et al. Intensified and protective $\mathrm{CD}^{+} \mathrm{T}$ cell immunity in mice with anti-dendritic cell HIV gag fusion antibody vaccine. J Exp Med. 2006;203(3):607-617.

49. Do Y, et al. Targeting of LcrV virulence protein from Yersinia pestis to dendritic cells protects mice against pneumonic plague. Eur J Immunol. 2010;40(10):2791-2796.

50. Cheong C, et al. Improved cellular and humoral immune responses in vivo following targeting of HIV Gag to dendritic cells within human anti- human DEC205 monoclonal antibody. Blood. 2010;116(19):3828-3838.

51. Tewari K, et al. Poly(I:C) is an effective adjuvant for antibody and multi-functional $\mathrm{CD} 4^{+} \mathrm{T}$ cell responses to Plasmodium falciparum circumsporozoite protein (CSP) and $\alpha$ DEC-CSP in non human primates. Vaccine. 2010;28(45):7256-7266.

52. Pantel A, et al. A new synthetic TLR4 agonist, GLA, allows dendritic cells targeted with antigen to elicit Th1 T-cell immunity in vivo. Eur J Immunol. 2012;42(1):101-109.

53. Ruane $\mathrm{D}$, et al. A dendritic cell targeted vaccine induces long-term HIV-specific immunity within the gastrointestinal tract. Mucosal Immunol. 2016;9(5):1340-1352.

54. Lakhrif Z, et al. Targeted delivery of toxoplasma gondii antigens to dendritic cells promote immunogenicity and protective efficiency against toxoplasmosis. Front Immunol. 2018;9:317.

55. Dhodapkar MV, et al. Induction of antigen-specific immunity with a vaccine targeting NY-ESO-1 to the dendritic cell receptor DEC-205. Sci Transl Med. 2014;6(232):232ra51.

56. Flynn BJ, et al. Immunization with HIV Gag targeted to dendritic cells followed by recombinant New York vaccinia virus induces robust T-cell immunity in nonhuman primates. Proc Natl Acad Sci USA. 2011;108(17):7131-7136.

57. Draper SJ, et al. Recombinant viral vaccines expressing merozoite surface protein-1 induce antibody- and T cell-mediated multistage protection against malaria. Cell Host Microbe.
2009;5(1):95-105.

58. Tapia MD, et al. Use of ChAd3-EBO-Z Ebola virus vaccine in Malian and US adults, and boosting of Malian adults with MVA-BN-Filo: a phase 1, single-blind, randomised trial, a phase $1 \mathrm{~b}$, open-label and double-blind, dose-escalation trial, and a nested, randomised, double-blind, placebo-controlled trial. Lancet Infect Dis. 2016;16(1):31-42.

59. Mensah VA, et al. Safety and Immunogenicity of Malaria Vectored Vaccines Given with Routine Expanded Program on Immunization Vaccines in Gambian Infants and Neonates: A Randomized Controlled Trial. Front Immunol. 2017;8:1551.

60. Coughlan L, et al. Heterologous two-dose vaccination with Simian adenovirus and poxvirus vectors elicits long-lasting cellular immunity to influenza virus A in healthy adults. EBioMedicine. 2018;29:146-154.

61. Luzuriaga K, Sullivan JL. Infectious mononucleosis. N Engl JMed. 2010;362(21):1993-2000.

62. Grässer FA, et al. Monoclonal antibodies directed against the Epstein-Barr virus-encoded nuclear antigen 1 (EBNA1): immunohistologic detection of EBNA1 in the malignant cells of Hodgkin's disease. Blood. 1994;84(11):3792-3798.

63. Hill AV, et al. Prime-boost vectored malaria vaccines: progress and prospects. Hum Vaccin. 2010;6(1):78-83.

64. Romao S, et al. Autophagy proteins stabilize pathogen-containing phagosomes for prolonged MHC II antigen processing. J Cell Biol. 2013;203(5):757-766 